\title{
GUIDE POUR L'ANALYSE STATISTIQUE DES ECHANTILLONS D'INVERTÉBRÉS BENTHIQUES
}

\author{
par J.-M. Ellotot ${ }^{1}$ et H. DÉcamps ${ }^{2}$.
}

\begin{abstract}
La planification de l'échantillonnage est envisagée dans le cas d'inventaires faunistiques extensifs et d'études quantitatives intensives. Des méthodes sont données pour décrire un échantillon en termes statistiques. La dispersion spatiale d'une population est discutée et les types fondamentaux de distribution décrits. Des tests sont présentés pour définir la dispersion au hasard, régulière ou contagieuse d'une population. Les problèmes d'estimation des nombres par unité de surface ou de volume sont exposés, ainsi que les méthodes de calcul des limites de confiance de ces estimations. Les méthodes paramétriques et non-paramétriques de comparaison quantitative de deux échantillons sont décrites et les utilisations du $\chi^{2}$ et des tables de contingence discutées. Une dernière partie est consacrée aux problèmes posés par l'échantillonnage sur le terrain et le sous-échantillonnage au laboratoire.
\end{abstract}

\section{A guide to statistical methods for the analysis of samples of benthic invertebrates}

The problems of planning a sampling programme for extensive faunal surveys and intensive quantitative studies are discussed. Methods are given for describing a sample in statistical terms. The spatial dispersion of a population is discussed, and the basic types of spatial distribution are described. Methods are given to test if the dispersion of a population is random, regular or contagious. The problems of estimating numbers per unit area or per unit volume are discussed, and methods are given to calculate confidence limits for these estimates. Both parametric and non-parametric methods for the quantitative comparison of two samples are described, and the uses of $\chi^{2}$ and contingency tables are discussed. A final section briefly discusses the problems of sampling in the field and sub-sampling in the laboratory.

1. Introduction ........................... 80

2. Planification du programme d'échantillonnage ........... 81

2.1. Inventaires faunistiques $\ldots \ldots \ldots \ldots \ldots \ldots \ldots \ldots \ldots, 81$

2.2. Etudes quantitatives .................. 82

2.2.1. Échantillons et populations ........... 83

2.2.2. Moyenne arithmétique et variance ....... 83

3. Dispersion spatiale d'une Population ................ 85

3.1. Distribution au hasard $\ldots \ldots \ldots \ldots \ldots \ldots \ldots \ldots \ldots .86$

3.1.1. Test de conformité avec une loi de Poisson .. 87

3.2. Distribution régulière $\ldots \ldots \ldots \ldots \ldots \ldots \ldots \ldots \ldots \ldots .90$

1. Freshwater Biological Association, Ferry House, Far Sawrey, Ambleside, Westmorland, England.

2. Laboratoire d'Hydrobiologie, Université Paul-Sabatier, 118, route de Narbonne, 31077 Toulouse Cedex. France. 
3.3. Distributions contagieuses $\ldots \ldots \ldots \ldots \ldots \ldots \ldots \ldots$

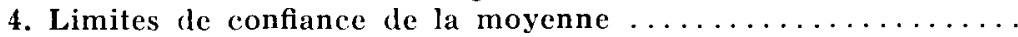

4.1. Limites de confiance de la moyenne d'un grand échantillon $(n>30)$

91

4.2. Limites de conflance de la moyenne d'un petit échantillon $(n<30)$ tiré d'une loi de Poisson (distribution au

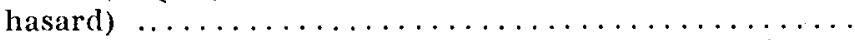

4.3. Limites de confiance de la moyenne d'un petit échantillon $(n<30)$ tiré d'une distribution contagieuse ....

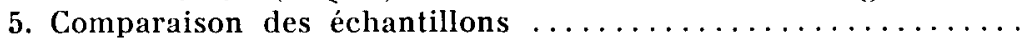

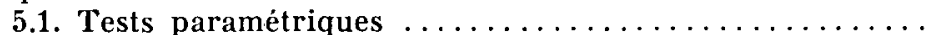

5.1.1. Comparaison des moyennes de deux grands échantillons $(n>50)$ : test de l'écart normal

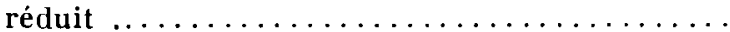

5.1.2. Comparaison des moyennes de deux petits échantillons $(n<50)$ tirés d'une loi de Poisson (distribution au hasard) $\ldots \ldots \ldots \ldots \ldots \ldots \ldots$

5.1.3. Comparaison des moyennes de deux petits échantillons $(n<50)$ tirés d'une distribution contagieuse : test $\mathrm{F}$ et test $t \ldots \ldots \ldots \ldots \ldots$

5.2. Méthodes non-paramétriques $\ldots \ldots \ldots \ldots \ldots \ldots \ldots$

5.2.1. Comparaison de deux échantillons : test $U$ de

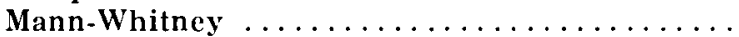

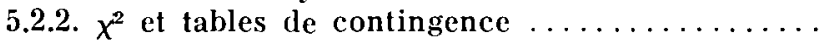
93 94 96 98 100

101

6. Quelques problèmes posés par l'échantillonnage sur le terrain

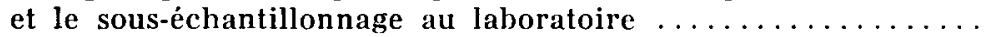

\section{INTRODUCTION}

L'échantillonnage de la faune du fond des eaux douces est généralement cntrepris pour connaître les espèces présentes, ainsi que la variation de leur densité dans le temps et dans l'espace. L'écologiste doit d'abord choisir une méthode convenable de prélèvement quantitatif (voir les descriptions d'appareils de Welch 1948, Macan 1958, Albrecht 1959, Cummins 1962, Southwood 1966, Schwoerbel 1970, Edmondson et Winberg 1971, Lamotte et Bourlière 1971). Il doit ensuite identifier les organismes, de préférence jusqu'à l'espèce (voir les clés de plusieurs groupes d'invertébrés aquatiques parues dans les Scientific Publications de la F.B.A. pour la Grande-Bretagne, dans les Annales de Limnologie pour la France, Bertrand 1954, et Macan 1959 pour une "clé des autres clés »). Enfin, les individus capturés doivent être dénombrés, et le procédé d'analyse choisi.

Cet article constitue un guide pratique pour l'analyse statistique des échantillons d'invertébrés benthiques. Il est basé sur l'exposé plus détaillé d'Elliott (1971). Cette analyse a pour but (1) de connaître la distribution du benthos, (2) d'estimer la population totale d'une surface donnée, (3) d'interpréter les différences quantitatives entre échantillons. Mais les méthodes statistiques ne sauraient 
tenir lieu d'opération de sauvetage. Elles doivent être considérées dès l'élaboration du programme d'échantillonnage. Cette planification est essentielle pour adapter les techniques de laboratoire ou de terrain aux objectifs recherchés, de même que pour fonder scientifiquement le choix des hypothèses et l'élaboration des estimations. Dans toutes les branches de l'écologie, l'époque des prélèvements faits sans plan préétabli est révolue. En hydrobiologie comme ailleurs, le naturaliste doit s'en convaincre s'il tient à exploiter correctement son travail de terrain.

Plusieurs des méthodes présentées ici font appel aux tables de Pearson et Hartley (1966). Dans le texte, les références à des ouvrages plus complets concernent des manuels de langue anglaise. En français, plusieurs ouvrages classiques sur l'application des méthodes statistiques en biologie pourront également être consultés (par exemple Lamotte 1971, Lison 1958, Vessereau 1960).

\section{Planification DU PROgRamme D'EchantillonNagli}

Il importe de bien définir les buts poursuivis et la surface à échantillonner car ces considérations déterminent la fréquence des prélèvements. L'échantillonnage peut ainsi être hebdomadaire dans l'étude détaillée d'un cycle vital, annuel lors d'un examen général. La plupart des investigations relèvent soit d'inventaires faunistiques extensifs, soit d'études quantitatives intensives.

\subsection{Inventaires faunistiques.}

L'inventaire d'une grande étendue (par exemple un lac, une rivière, etc.) précède généralement une étude quantitative, mais peut constituer une fin en soi. L'ensemble de l'aire à étudier est habituellement divisée en sections égales. Une ou plusieurs stations sont choisies, si possible au hasard, dans chacune de ces sections. L'aire à étudier peut également être divisée en biotopes distincts (par exemple substrat pierreux, vase, mousses, etc.), et les stations sont choisies au hasard dans chacun de ces biotopes.

Un inventaire faunistique consiste principalement à recenser les espèces présentes et à estimer leur abondance relative dans chaque station. Comme il est important de capturer les espèces rares aux différentes stations, chaque prélèvement doit recouvrir une grande surface. Une méthode simple consiste à recueillir la faune au moyen d'un filet-troubleau pendant un temps fixé à l'avance. Elle peut être utilisée en eau courante ou stagnante. En eau courante, on retourne les pierres à la main ou remue le fond avec le pied; le courant entraîne le matériel dans le filet de récolte. L'opérateur se déplace lentement vers l'amont pendant deux minutes et couvre ainsi une grande surface. En eau stagnante, une technique sem- 
blable peut être utilisée au bord d'un lac, sur substrat pierreux. On soulève et nettoie les pierres à l'ouverture d'un filet rapidement glissé vers l'avant. L'opérateur se déplace lentement le long du bord pendant deux minutes. Plusieurs récoltes peuvent être nécessaires lorsqu'une station recouvre une grande surface.

Ces techniques simples présentent quelques avantages :

1. Elles n'exigent pas d'appareils très élaborés;

2. Elles permettent la capture d'une grande partie des espèces présentes;

3. Elles fournissent des données comparables, notamment lorsque le biotope et l'opérateur sont les mêmes pour tous les échantillons. Les pourcentages peuvent ainsi être comparés entre stations.

Elles présentent aussi des inconvénients :

1. Elles ne peuvent pas être utilisées en eau profonde;

2. La faune est prélevée pendant un temps donné et non sur une surface fixe. On ne peut donc pas estimer les nombres par unité de surface : les comparaisons entre échantillons sont limitées à l'abondance relative. De plus, l'importance de chaque espèce est exprimée en pourcentage du nombre total, et par suite influencée par la composition de l'échantillon. Ces comparaisons n'ont donc qu'une portée limitée.

Des prélèvements quantitatifs peuvent être préférés, en utilisant l'un des nombreux échantillonneurs construits à cet effet. La surface des relevés (= unités d'échantillonnage) est alors connue, mais cette surface est généralement petite. Il convient donc de fixer le nombre de relevés nécessaires pour que l'échantillon contienne la plupart des espèces présentes. Si les relevés sont pris sur un transect correspondant à un gradient écologique quelconque (profondeur, courant, ...), les espèces ont, pour la plupart, une forte chance d'être capturées au moins une fois. Quand les espèces peuvent être identifiées sur le terrain, les relevés sont pris au hasard et le nombre d'espèces nouvelles est noté à chaque relevé. Le prélèvement est arrêté quand trois relevés successifs n'ajoutent pas d'espèces à la liste totale. Le nombre de relevés nécessaires dépendra, pour chaque station, de la diversité et de la dispersion de la faune du fond. Gaufin et al. (1956) ont proposé une formule pour calculer le nombre moyen d'espèces nouvelles apportées par le kème relevé ( $k=1$ ou plus). Harris (1957) a présenté une formule pour calculer les erreurs-standard de telles estimations.

\subsection{Etudes quantitatives.}

Les méthodes décrites dans la suite de cet article concernent des études quantitatives et supposent la connaissance des nombres par 
unité d'aire. Il est souvent impossible de faire pour toutes les espèces une estimation complète el précise sur une grande surface. Aussi, la plupart des études quantitatives se limitent-elles à un petit nombre d'espèces sur une grande surface ou à un grand nombre d'espèces sur une petite surface. Cette surface doit être clairement définie, par exemple une certaine section d'un lac ou d'une rivière.

\subsection{1. Échantillons et populations.}

Il est rarement possible de compter tous les invertébrés d'une Population, d'où la nécessité d'un échantillonnage. Les deux acceptions du terme «population» sont distinguées ici par une majuscule $\mathbf{P}$ (sens écologique) et une minuscule p (sens statistique). En écologie, une «Population» est le nombre total d'individus d'une espèce présents dans une aire donnée : ensemble ou section d'un lac, d'une rivière. En statistique, tont ensemble de valeurs est une «population», et par suite l'ensemble des relevés qui, par juxtaposition, composent une aire est « une population de relevés». Par exemple, la population pour un lac ou une rivière est l'aire totale du fond, et cette aire est divisée en relevés d'égale dimension. Chaque opération d'un échantillonneur extrait un relevé du fond et la dimension de ce relevé dépend du type d'appareil utilisé. Les relevés doivent être distincts, sans emboîtements, et former l'ensemble de la population lorsqu'ils sont juxtaposés. Comme il est rarement possible d'extraire tous les relevés d'une population, seuls certains sont choisis et supposés représentatifs de l'ensemble de la population. Ce groupe de relevés (= unités d'échantillonnage) forme un "échantillon». Dans cet échantillon, les relevés ont la même dimension, sont pris pendant un court laps de temps, et sont généralement choisis au hasard dans l'ensemble de la population de relevés. L'information de base est toujours obtenue par dénombrement des individus de tous les relevés de l'échantillon. Le «nombre» d'individus par relevé est donc la variable à étudier.

\subsubsection{MOYENNE ARITHMÉTIQUe FT VARIANCE.}

Dans la figure 1, les nombres obtenus par une série de relevés sont représentés par $\mathrm{x}_{1}, \mathrm{x}_{2}, \mathrm{x}_{3} \ldots \mathrm{x}_{\mathrm{n}}$, il $\mathrm{y}$ a $\mathrm{n}$ relevés dans l'échantillon pris au hasard, et la «moyenne arithmétique» de l'échantillon est représentée par $\bar{x}$. Plusieurs « moyennes » existent pour une série de nombres, par exemple médiane, mode, moyenne arithmétique, moyenne harmonique, moyenne géométrique, et la valeur moyenne utilisée doit toujours être précisée. La moyenne arithmé- 
tique est probablement la plus utile et sera la plus employée dans cet exposé.

Il apparaît toujours une certaine variabilité dans les nombres d'un échantillon pris au hasard. Cette variabilité est due en partie aux défauts de la technique d'échantillonnage, par exemple certains invertébrés peuvent passer à travers les mailles du filet, s'échapper de l'échantillonneur, ou être manqués (voir Macan 1958,
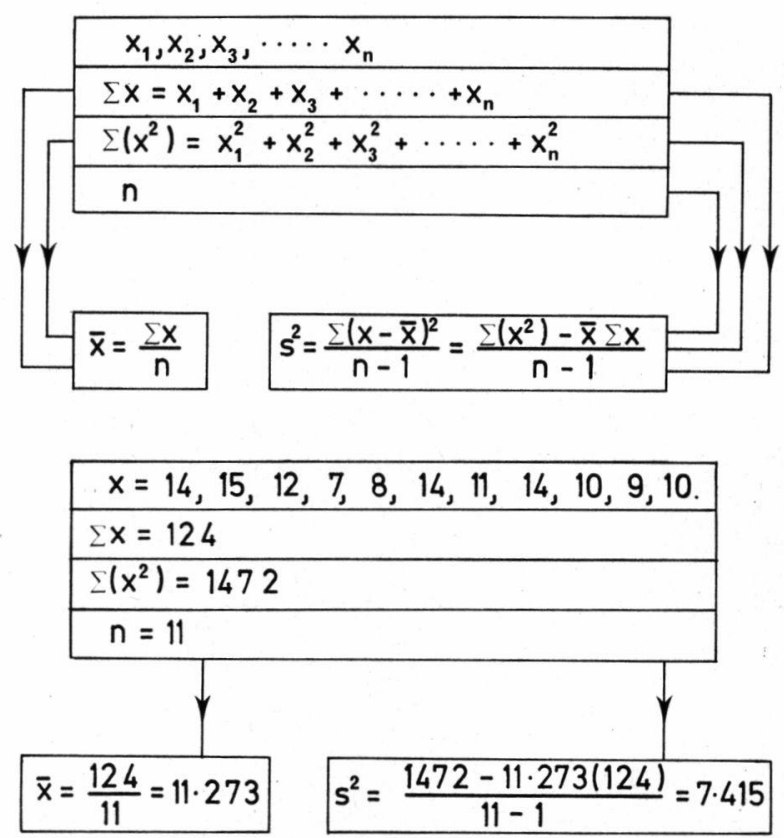

FIG. 1. - Calcul de la moyenne arithmétique $(\overline{\mathrm{x}})$ et de la variance $\left(\mathrm{s}^{2}\right)$.

TABLE I. - Comparaison des symboles utilisés pour les estimations de l'échantillon et les paramètres de la population.

Estimations
de l'échantillon de la population

Moyenne arithmétique

Variance

Ecart-type

Moyenne et variance d'une loi de Poisson

Nombre de relevés

$\begin{array}{cc}\overline{\mathrm{x}} & \mu \\ \mathrm{s}^{2} & \sigma^{2} \\ \mathrm{~s} & \sigma \\ \mathrm{m} & \lambda \\ \mathrm{n} & \end{array}$


Albrecht 1959 et Cummins 1962). Il est souvent impossible d'évaluer «l'erreur»d'un échantillonneur, mais cette erreur sera généralement du même ordre pour tous les relevés. Si l'efficacité de l'échantillonneur est normale, la principale cause de variation viendra de la distribution des invertébrés sur le fond du lac ou du cours d'eau. Cette variation est mesurée par la «variance» $\left(s^{2}\right)$ de l'échantillon.

Nous pouvons donc caractériser un échantillon par sa valeur moyenne (moyenne arithmétique $\bar{x}$ ) et par une mesure de la dispersion des nombres (variance $\mathrm{s}^{2}$ ). Ces « estimations» sont calculées à partir de l'échantillon (fig. 1) et correspondent aux «paramètres » de la population globale. Les paramètres de la population sont généralement représentés par des lettres grecques, les estimations de l'échantillon par des lettres romaines (Table I).

\section{DISPERSION SPATIALE D'UNE POPULATION}

La «dispersion spatiale d'une Population 》 décrit la distribution dans l'espace des individus au sein de la Population (sens écologique, voir $\$$ 2.2.1.). Cette dispersion varie dans le temps, notamment pou les stades successifs d'une même espèce. La dispersion d'une Population revêt une importance pratique car elle règle l'analyse des échantillons et le programme d'échantillonnage.

Les individus d'une Population peuvent montrer trois types fondamentaux de distribution dans l'espace :

1) distribution au hasard,

2) distribution régulièrc (= sous-dispersion, ou distribution uniforme, ou égale),

3) distribution contagieuse (= sur-dispersion, ou dispersion en groupes, ou en agrégats).

Les termes régulière et contagieuse peuvent être critiqués, mais ce sont les plus fréquemment utilisés; ils prêtent moins à confusion que ceux entre parenthèses. Il faut remarquer que les trois types de distribution peuvent se recouvrir, par exemple une distribution contagieuse peut résulter de groupes distribués au hasard, avec des individus régulièrement distribués dans chaque groupe.

La dispersion d'une Population détermine les relations entre la variance $\left(\sigma^{2}\right.$ estimée par $s^{2}$ ) et la moyenne arithmétique ( $\mu$ estimée $\operatorname{par} \overline{\mathbf{x}})$ :

1) distribution au hasard : variance égale à la moyenne $\left(\sigma^{2}=\mu\right)$;

2) distribution régulière : variance inférieure à la moyenne $\left(\sigma^{2}<\mu\right)$; 
3) distribution contagieuse : variance supérieure à la moyenne $\left(\sigma^{2}>\mu\right)$.

Ces relations orientent le choix d'un test convenable pour étudier la dispersion spatiale d'une Population (§ 3.1.1.).

\subsection{Distribution au hasard.}

D'ordinaire, la première hypothèse à considérer est celle d'une distribution au hasard. Dans une telle distribution, tous les individus ont une chance égale d'occuper un point quelconque du fond, et la présence d'un individu n'influence pas la position d'un individu voisin. Le fait manquant d'une distribution au hasard est l'absence de tout système: certains individus sont groupés, d'autres également espacés; certains sont très proches, d'autres très espacés.

La conformité avec une loi de Poisson est le test utilisé pour une distribution au hasard. Plusieurs ouvrages décrivent en détail les propriétés de la loi de Poisson (par exemple Elliott 1971, page 18). Une propriété importante est que la variance et la moyenne arithmétique sont égales $\left(\sigma^{2}=\mu\right)$ et toutes deux égales au paramètre $\lambda$ de Poisson, estimé par $\mathrm{m}\left(\mathrm{m}=\overline{\mathrm{x}}=\mathrm{s}^{2}\right)$.

Aucun test ne peut prouver qu'une distribution se fait au hasard. La conformité avec une loi de Poisson signifie seulement que l'hypothèse d'une distribution au hasard n'est pas contredite. Mais il est toujours possible que cette hypothèse soit fausse sans que cela puisse être vérifié. Aussi, avant d'en accepter l'hypothèse est-il important de considérer pour quelles raisons une espèce peut être distribuée au hasard. Il peut s'agir 1) soit de l'influence d'un facteur unique dont les valeurs sont elles-mêmes distribuées au hasard, 2 ) soit des effets du hasard lui-même. Les facteurs du milieu suivent rarement une distribution au hasard, ce qui rend la première explication peu vraisemblable. Exception possible, le comportement de ponte pourrait entraîner une distribution au hasard des premiers stades si les œufs étaient déposés isolément et au hasard. Mais beaucoup d'espèces déposent leurs œeufs par groupes. La première explication étant rejetée, la deuxième doit être considérée, avec comme corollaires : 1) les facteurs du milieu n'affectent pas, ou relativement peu, la dispersion de la Population, 2) les individus de la Population ne tendent aucunement à s'éviter ou à se rassembler. Il est difficile d'accepter ces propositions, et la seule conclusion est qu'une distribution non-au hasard existe mais ne peut pas être décelée par les techniques d'échantillonnage sur le terrain.

L'indication d'une distribution non-au hasard dépend de la densité de la Population et de la taille du relevé. Si la taille du 
relevé (c'est-à-dire du quadrat) est beaucoup plus grande ou beaucoup plus petite que la taille moyenne des groupes d'individus (ces groupes étant distribués régulièrement ou au hasard), la dispersion de la Population paraît au hasard, et le non-hasard n'est pas décelé. La plupart des échantillonneurs décèleront le non-hasard si le relevé est petit (environ $0,05 \mathrm{~m}^{2}$ au moins), mais une distribution contagieuse ne sera pas décelée, même avec un petit quadrat, s'il n'existe que quelques individus par groupe. En fait, la dispersion d'une Population est au hasard si la densilé de la Population est faible. Avec l'âge, une Population tend souvent à se distribuer au hasard, mais ce changement vient soit d'une diminution de la densité, soit de la division des grands groupes en plus petits.

En général, l'hypothèse d'une distribution au hasard convient donc aux Populations de faible densité, mais les implications de cette distribution doivent être soigneusement considérées lorsqu'il s'agit de l'appliquer à d'autres populations. Si l'hypothèse d'une distribution au hasard ne peut pas être rejetée, elle est acceptée car elle est la plus simple possible; en de nombreux cas, on ne trouve aucun avantage à tenter autre chose! Pratiquement, une distribution au hasard permet d'utiliser une loi de Poisson, avec ses méthodes relativement simples de calcul des limites de confiance et de comparaison des échantillons.

\subsubsection{Test de conformité avec une lot de Poisson.}

Dans une loi de Poisson, la variance et la moyenne étant égales, le rapport variance/moyenne ou «Indice de dispersion» (I) tend vers l'unité $\left(I=s^{2} / \bar{x}=1\right)$. En réalité, cet indice s'écarte souvent de l'unité et la signification des écarts est donnée par le calcul de $\chi^{2}=\mathbf{s}^{2}(n-1) / \bar{x}$ (voir fig. 2). La conformité avec une loi de Poisson est acceptée au seuil de probabilité $95 \%(\mathrm{P}>0,05)$ si la valeur de $\chi^{2}$ est comprise entre les seuils appropriés de signification à $5 \%$ pour $\mathrm{v}=\mathrm{n}-1$ degrés de liberté. Les seuils de signification sont donnés par des tables de $\chi^{2}$, et localisés dans les colonnes $Q=0,975$ et $Q=0,025$ pour $\mathrm{v}$ degrés de liberté (Table II).

Si l'échantillon est grand $(n>31)$, on suppose que $\sqrt{2 \chi^{2}}$ est distribué normalement autour de $\sqrt{2} \overline{\mathrm{v}-1}$ avec une variance unité. La variable normale réduite (d) est alors calculée (fig. 2), et la conformité avec une loi de Poisson est acceptée $(P>0,05)$ si la valeur absolue de d (c'est-à-dire sans tenir compte du signe) est inférieure à 1,96 . 
Table II. - Seuils de signification à $5 \%(Q=0,975$ et 0,025$)$ pour un test $\chi^{2}$ de conformité avec une loi de Poisson (voir $\$ 3.1 .1$ ), et valeur $5 \%$ $(Q=0,050)$ pour les tests $\chi^{2}$ d'ajustement (voir $\$ 5.2 .2$.).

\begin{tabular}{|c|c|c|c|}
\hline $\mathbf{v}$ & $\begin{array}{c}\text { Niveau } \\
\text { inférieur } \\
(Q=0,975)\end{array}$ & $\begin{array}{c}\text { Niveau } \\
\text { supérieur } \\
(Q=0,025)\end{array}$ & $\begin{array}{l}\text { Valeur } 5 \% \\
(Q=0,050)\end{array}$ \\
\hline 1 & $<0.01$ & 5.02 & 3.84 \\
\hline 2 & 0.05 & 7.38 & 5.99 \\
\hline 3 & 0.22 & 9.35 & 7.81 \\
\hline 4 & 0.48 & 11.14 & 9.49 \\
\hline .5 & 0.83 & 12.83 & 11.07 \\
\hline 6 & 1.24 & 14.45 & 12.59 \\
\hline 7 & 1.69 & 16.01 & 14.07 \\
\hline 8 & 2.18 & 17.53 & 15.51 \\
\hline 9 & 2.70 & 19.02 & 16.92 \\
\hline 10 & 3.25 & 20.48 & 18.31 \\
\hline 11 & 3.82 & 21.92 & 19.68 \\
\hline 12 & 4.40 & 23.34 & 21.03 \\
\hline 13 & 5.01 & 24.74 & 22.36 \\
\hline 14 & 5.63 & 26.12 & 23.68 \\
\hline 15 & 6.26 & 27.49 & 25.00 \\
\hline 20 & 9.59 & 34.17 & 31.41 \\
\hline 25 & 13.12 & 40.65 & 37.65 \\
\hline 30 & $16.7 y$ & 46.98 & 43.77 \\
\hline
\end{tabular}

Les différences avec une loi de Poisson, donc avec une distribution au hasard, peuvent se produire dans deux directions. En effet, la valeur de $\chi^{2}$ peut être :

1) plus petite que présumée $\left(x^{2}<\right.$ valeur prévue pour $Q=0,975$, ou $d>1,96$ avec un signe positif) et l'on peut donc s'attendre à une distribution régulière $\left(\mathrm{s}^{2}<\mathrm{x}\right)$,

2) plus grande que présumée $\left(\chi^{2}>\right.$ valeur prévue pour $Q=0,025$, ou $d>1,96$ avec un signe positif) et l'on peut donc s'attendre à une distribution contagieuse $\left(\mathrm{s}^{2}>\overline{\mathrm{x}}\right)$.

Si l'échantillon est grand, le résultat de ce test doit être vérifié par le test $\chi^{2}$ d'ajustement (voir Elliott 1971, page 44).

Quatre exemples d'application du test $\chi^{2}$ (rapport variance à la moyenne) sont donnés dans la figure 2 pour des échantillons pris au hasard, petits $(2 \mathrm{~A}$ et $2 \mathrm{~B}$ ) et grands $(2 \mathrm{C}$ et $2 \mathrm{D})$. Dans le premier exemple, le $\chi^{2}(=6,6)$ est compris entre les seuils de signification à $5 \%$, c'est-à-dire $3,25(\mathrm{Q}=0,975)$ et $20,48(\mathrm{Q}=0,025)$ pour $\mathrm{v}=10$ degrés de liberté. La conformité avec une loi de Poisson est donc acceptée au seuil de probabilité $95 \%(\mathrm{P}>0,05)$, et l'hypothèse d'une distribution au hasard n'est pas écartée; autrement dit, il est possible que la dispersion de la Population soit au hasard. Dans l'exemple $2 \mathrm{~B}$, le $\chi^{2}(=220,0)$ est supérieur au seuil 


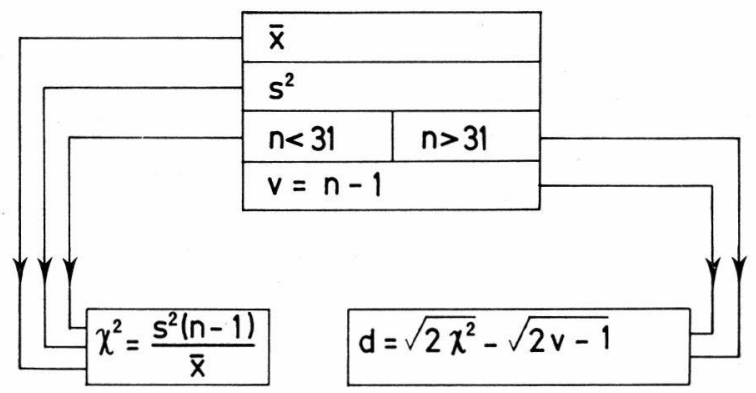

$2 A$

\begin{tabular}{|l|}
\hline $\bar{x}=11 \cdot 273$ \\
\hline$s^{2}=7 \cdot 415$ \\
\hline$n=11$ \\
$v=10$ \\
$\chi^{2}=\frac{7 \cdot 415(10)}{11 \cdot 273}=6.6$ \\
\hline
\end{tabular}

2C

\begin{tabular}{|l|}
\hline $\bar{x}=10 \cdot 125$ \\
\hline$s^{2}=8.592$ \\
\hline$n=80$ \\
\hline$v=79$ \\
$\chi^{2}=\frac{8.592(79)}{10 \cdot 125}=67.0$ \\
$\downarrow$ \\
$d=\sqrt{134}-\sqrt{157}=-0.9$ \\
\hline
\end{tabular}

$2 B$

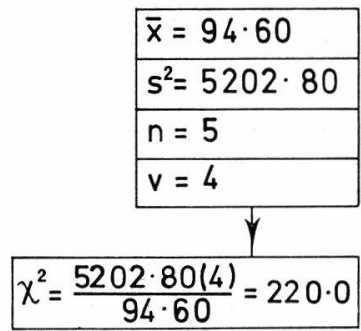

2D

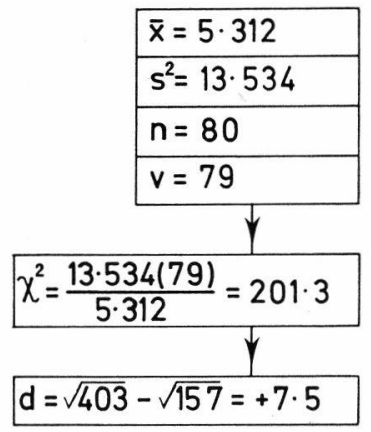

FIG. 2 - Test $\chi 2$ (rapport variance à la moyenne) pour conformité avec une loi de Poisson. La conformité avec une loi de Poisson est acceptée pour les exemples $2 \mathrm{~A}$ (petit échantillon) et $2 \mathrm{C}$ (grand échantillon), et rejetée pour $2 \mathrm{~B}$ (petit échantillon) et $2 \mathrm{D}$ (grand échantillon).

de signification $5 \%$, c'est-à-dire $11,14(\mathrm{Q}=0,025)$ pour $\mathrm{v}=4$ degrés de liberté. La conformité avec une loi de Poisson est donc rejetée au seuil de probabilité 95\% $(\mathrm{P}<0,05)$. La valeur élevée du $\chi^{2}$ indique que la dispersion spatiale de la Population est contagieuse : les animaux se présentent par groupes. Ces deux échan- 
tillons ont été pris au hasard dans une même section de cours d'eau, mais en des mois différents. Le grand écart observé entre les $\chi^{2}$ montre combien le mode de dispersion d'une même espèce peut changer au cours de l'année. Pour les grands échantillons, d est inférieur à 1,96 dans l'exemple $2 \mathrm{C}$. La conformité avec une loi de Poisson est donc acceptée $(P>0,05)$ et l'hypothèse d'une distribution au hasard n'est pas écartée; autrement dit, il est possible que la dispersion de la Population soit au hasard. Dans l'exemple $2 \mathrm{D}$, d est supérieur à 1,96 , avec un signe positif. La conformité avec une loi de Poisson est donc rejetée $(\mathrm{P}<0,05)$ et la dispersion de la Population est nettement contagieuse $\left(\mathbf{s}^{2}>\overline{\mathrm{x}}\right.$, d'où $\left.\sigma^{2}>\mu\right)$. Par suite, le test de $\chi^{2}$ indique non seulement la conformité avec une loi de Poisson, mais aussi le mode de dispersion de la Population : au hasard, régulier ou contagieux.

\subsection{Distribution régulière.}

La dispersion d'une Population est régulière quand les individus sont relativement nombreux tout en s'écartant les uns des autres. Le nombre d'individus par relevé est alors voisin du maximum possible, et la variance de la population inférieure à la moyenne $\left(\sigma^{2}<\mu\right)$. Le caractère essentiel d'une distribution régulière est l'espacement uniforme des individus de la Population. A la limite, dans une distribution parfaitement régulière, les individus sont équidistants les uns des autres.

Un comportement de territoire conduit souvent à l'espacement uniforme des individus; et par suite, la dispersion d'invertébrés sédentaires peut être régulière sur une petite surface, par exemple les larves de Chironomides vivant dans des tubes, les larves de Trichoptères construisant un filet, les larves de Simuliidae. Bien que, pour la plupart des espèces, la distribution soit irrégulière à l'échelle d'une grande surface, une certaine régularité peut apparaître au sein des groupes d'individus. Par exemple, un groupe de larves de Baetis rhodani peut montrer une distribution régulière sur la face supérieure d'une pierre pendant la nuit. Ces larves broutent les algues, et la distribution régulière est probablement due à ce que chaque larve possède un territoire de broutage défini.

Par suite, une distribution régulière décrira rarement la dispersion d'une Population à l'échelle d'une grande surface; elle la décrira quelquefois à l'échelle d'une surface plus réduite. L'étude de la microdispersion exige des techniques spéciales, par exemple les méthodes du plus proche voisin (Southwood 1966, page 40), dont la description dépasse le cadre de cet exposé. Si les animaux sont visibles, leur distribution peut être photographiée (par exemple Heywood et Edwards 1961) ou marquée sur le fond transpa- 
rent d'une boîte de visée. Edgar et Meadows (1969) ont ainsi utilisé les méthodes du plus proche voisin pour l'étude de la distribution spatiale des larves de Chironomus riparius et exposé en détail leurs méthodes statistiques.

\subsection{Distributions contagieuses.}

La distribution spatiale d'une Population est rarement au hasard ou régulière. Elle est, dans la plupart des cas, du type contagieux, avec la variance significativement plus grande que la moyenne. Des groupes ou agrégats définis d'individus apparaissent toujours dans une distribution contagicuse, mais l'aspect final varie considérablement. La fréquence des distributions contagieuses n'est pas surprenante car beaucoup de facteurs sont distribués inégalement. De plus, certaines espèces ont tendance à s'agréger et présentent ainsi une distribution contagieuse sans influence externe. La dispersion finale dépend de la taille des groupes, de la distance entre groupes, de la distribution spatiale des groupes, et de la distribution spatiale des individus à l'intérieur des groupes. Un aspect courant est celui de taches de forte densité (essaims) sur un fond général de faible densité. Mais, dans le même habitat, des espèces différentes présentent des distributions contagieuses différentes, et le mode de dispersion d'une même espèce peut varier sur une petite surface. Il existe donc divers types de distributions contagieuses et il est difficile de trouver une distribution mathématique ajustée à tous ces types. Plusieurs modèles mathématiques ont été proposés. Le plus utile d'entre eux est probablement la distribution binomiale négative. La formation et les propriétés de la binomiale négative et d'autres modèles mathématiques des distributions contagieuses sont décrites en détail par Elliott (1971, chapitre 5), et sont trop complexes pour être incluses dans cet exposé élémentaire. Ces modèles s'appliquent habituellement à de grands échantillons $(n>30)$ et sont des plus intéressants lorsqu'on se propose d'étudier la dispersion d'une Population en détail. Dans des études quantitatives sur petits échantillons, il est important de reconnaître une distribution contagieuse par un test $\chi^{2}$ (rapport variance/ moyenne) et de prendre les précautions convenables dans l'analyse des données.

\section{LIMITES DE CONFIANCE DE LA MOYENNE}

Nous avons noté (§ 2.2.2.) que la moyenne arithmétique $(\mu)$ d'une population était estimée par la moyenne arithmétique ( $x$ ) d'un échantillon de cette population. Cette moyenne estimée sert 
souvent à déterminer les nombres totaux de l'aire étudiée. Il est donc important de connaître la précision de l'estimation.

Par exemple, le nombre moyen de larves dans $100 \mathrm{~cm}^{2}$ est 10,125 . La moyenne estimée de la population est donc 10,125 larves par $100 \mathrm{~cm}^{2}$. Si les relevés sont pris sur une section de cours d'eau dont l'aire totale est de $30 \mathrm{~m}^{2}\left(=300000 \mathrm{~cm}^{2}\right)$, l'estimation du nombre total de larves dans l'aire étudiée est :

$$
(300000 / 100) \quad 10,125=30375 .
$$

Il serait bien étonnant que cette estimation concorde avec le nombre réel de larves présentes dans l'aire étudiée, d'où la nécessité de préciser «l'erreur » de la moyenne estimée de la population.

Au moment de l'échantillonnage, la moyenne vraie $(\mu)$ de la population n'a qu'une valeur, tandis que la moyenne $(\bar{x})$ de l'échantillon varie d'un échantillon à l'autre. Par exemple, six échantillons peuvent avoir comme moyennes : 9,51 - 10,74 - 9,82 $10,20-10,52$ et 10,13 ; chacune de ces valeurs peut être utilisée comme estimation de la moyenne inconnue de la population. S'il était possible de prélever un grand nombre d'échantillons de la même population et de ranger les moyennes de ces échantillons en une distribution de fréquences, cette dernière tendrait vers la normalité même si, dans chaque échantillon, les nombres suivaient des distributions non-normales, par exemple binomiale positive, Poisson, ou binomiale négative. Ce résultat important est résumé par le «théorème central limite »: les moyennes de grands échantillons pris au hasard $(n>30)$ dans la même population sont approximativement distribuées normalement avec une moyenne égale à la moyenne vraie de la population $(\mu)$ et une variance liée à la variance $\left(\sigma^{2}\right)$ de la population par la formule simple : variance des moyennes des échantillons $=\sigma^{2} / \mathrm{n}$. Il s'ensuit que l'écart-type de la distribution des moyennes (encore appelé «erreur-type» ou «erreur-standard» de la moyenne) est $\sqrt{\sigma^{2} / n}$; il est estimé par l'erreur-standard de l'échantillon : $\sqrt{\mathbf{s}^{2} / \mathbf{n}}$. En conséquence, l'estimation de $\mu$ est souvent écrite sous la forme $\bar{x} \pm$ erreur-standard $\left(\overline{\mathrm{x}} \pm \sqrt{\mathbf{s}^{2} / \mathrm{n}}\right)$. Lorsque la taille de l'échantillon (c'est-à-dire $n$ ) augmente, l'erreur standard (S.E.) diminue et la moyenne estimée de la population tend vers $\mu$.

Les «limites de confiance», souvent utilisées à la place de l'erreur-standard de la moyenne sont d'une interprétation plus facile. Elles définissent les valeurs supérieure et inférieure de l'intervalle dans lequel se tient la vraie moyenne de la population, par exemple $95 \%$ des limites de confiance indique qu'il existe 95 chances contre 5 (ou 19 contre 1 ) pour que la moyenne de la population se tienne entre ces limites. 


\subsection{Limites de confiance de la moyenne d'un grand échantillon (n $>30)$.}

Si un grand nombre de relevés forme l'échantillon ( $\mathbf{n}>30)$, et si ces relevés sont pris au hasard, le théorème central limite peut être appliqué. La moyenne de l'échantillon est alors l'une des moyennes possibles normalement distribuées autour de la moyenne vraie de la population. Dans une distribution normale, $95 \%$ des valeurs se tiennent dans un intervalle de 1,96 écartstypes de part et d'autre de la moyenne (voir manuels courants). Par suite, $95 \%$ des moyennes possibles des échantillons se trouvent dans un intervalle de $\mathbf{1 , 9 6}$ erreurs-standard de part et d'autre de la moyenne de la population, et les chances sont de 19 contre $1(\mathrm{P}=0,95)$ pour qu'une moyenne d'échantillon soit à l'intérieur de cet intervalle. Des chances semblables $(P=0,95)$ existent pour que la moyenne de la population $(\mu)$ soit à l'intérieur de l'intervalle $\mathbf{1 , 9 6}$ erreurs-standard de part et d'autre de la moyenne de l'échantillon $(\overline{\mathbf{x}})$; les limites de cet intervalle correspondent aux $95 \%$ des limites de confiance de la moyenne. La limite de confiance la plus basse est $\bar{x}-1,96$ erreurs-standard, et la limite supérieure est $\bar{x}+1,96$ erreurs-standard; ou plus exactement, les $95 \%$ des limites de confiance sont donnés par $\overline{\mathbf{x}} \pm \mathbf{t} \sqrt{\mathbf{s}^{2} / \mathbf{n}}$ où $\sqrt{\mathbf{s}^{2} / \mathbf{n}}$ est l'erreur-standard de l'échantillon, $t$ étant trouvé dans la «distribution du $t$ de Student». Le $t$ de Student doit toujours être utilisé quand la variance de la population $\left(\sigma^{2}\right)$, étant inconnue, doit être estimée par la variance de l'échantillon $\left(s^{2}\right)$. La valeur de $t$ dépend du nombre de degrés de liberté (n-1), et augmente régulièrement lorsque le nombre de degrés de liberté diminue. Avec de grands échantillons $(n>30), t=2$ est une bonne approximation pour les $95 \%$ des limites de confiance.

Un exemple du calcul des limites de confiance est donné dans la figure 3 (exemple $3 \mathrm{~A}$ ), et ces limites peuvent être utilisées pour estimer la population totale de l'aire étudiée. Les limites de confiance $10,125 \pm 0,612$ indiquent qu'il $y$ avait entre 9,51 et 10,74 larves d'Ephémères $/ 100 \mathrm{~cm}^{2}$ (le relevé était un quadrat de $10 \mathrm{~cm}$ de côté). Nous avons estimé (début du $\S 4$ ) à 30375 le nombre de larves présentes dans une section de $30 \mathrm{~m}^{2}$ de ruisseau. Il est maintenant possible de calculer les $95 \%$ des limites de confiance de cette estimation : 28539 et 32211 . Par suite, le nombre total dans $30 \mathrm{~m}^{2}$ de ruisseau est probablement $(\mathrm{P}=0,95)$ entre 28539 et 32211 larves. Une estimation plus précise ne peut être obtenue qu'en augmentant le nombre de relevés de l'échantillon. Il est illusoire d'indiquer pour seule valeur 30375 larves, nombre obtenu à partir de la moyenne de l'échantillon. 


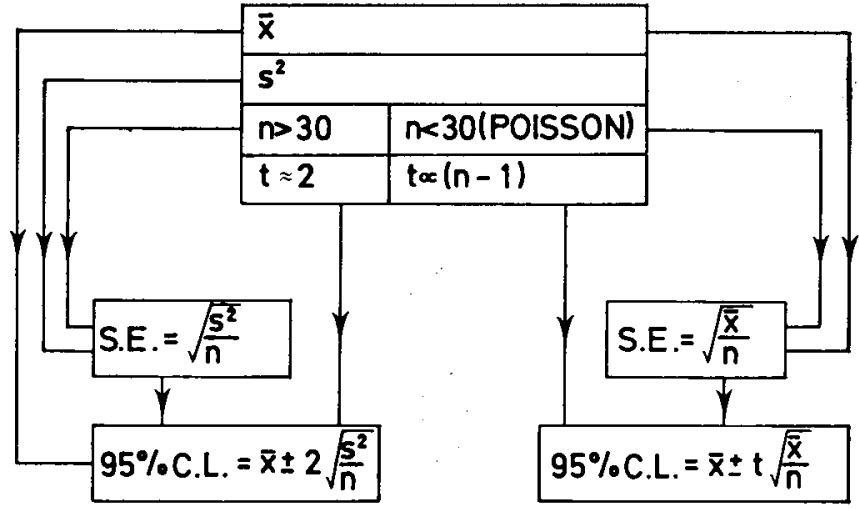

$3 \mathbf{A}$

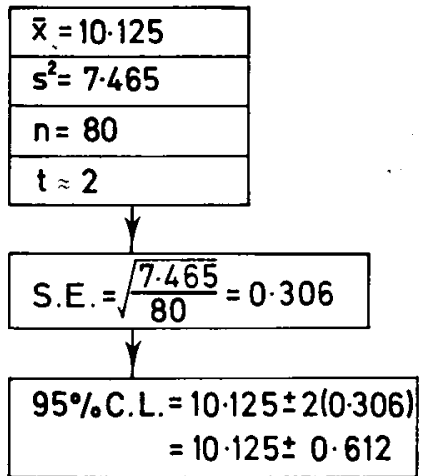

$3 \mathrm{~B}$

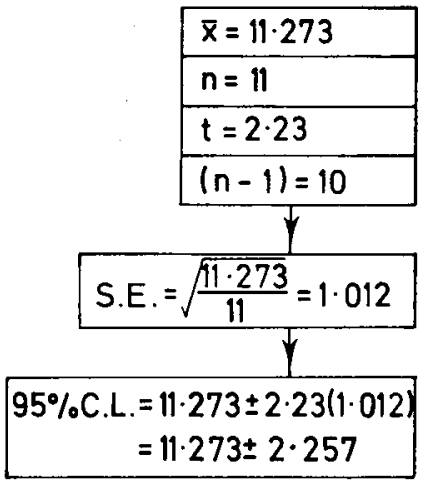

Fig. 3. - Calcul de l'erreur-standard (S. E.) et des $95 \%$ des limites de confiance (C. L.) de la moyenne pour un grand échantillon (exemple $3 \mathrm{~A}$ ) et pour un petit échantillon $(n<30)$ tiré d'une loi de Poisson, c'est-à-dire une distribution au hasard (exemple $3 \mathrm{~B}$ ).

4.2. Limites de confiance de la moyenne d'un petit échantillon $(\mathbf{n}<30)$ tiré d'une loi de Poisson (distribution au hasard).

Le théorème central limite ne peut être appliqué à la plupart des petits échantillons $(n<30)$. Une exception existe pour la loi de Poisson quand le produit $\mathrm{nm}$ est plus grand que 30 (m est l'estimation du paramètre $\lambda$ de Poisson). Comme $\mathbf{x}=\mathbf{m}=\mathbf{s}^{2}$ dans la loi de Poisson (voir $\S 3.1$. ), $\bar{x}$ est la meilleure estimation à la fois de $\mathrm{m}$ et de $\mathrm{s}^{2}$; l'estimation de $\mathrm{m}$, avec son erreur-standard, est donc : $\overline{\mathrm{x}} \pm \sqrt{\overline{\mathrm{x}} / \mathrm{n}}$. Par suite, les $95 \%$ des limites de confiance pour l'estimation de la moyenne $\mu$ de la population $\left(\mu=\lambda=\sigma^{2}\right)$ sont : $\bar{x} \pm t \sqrt{x / n}, t$ étant indiqué par la table III. 
Un exemple de calcul des limites de confiance est donné dans la figure 3 (exemple $3 \mathrm{~B}$ ). Les limites de confiance 11,273 $\pm 2,257$ indiquent que la vraie moyenne de la population $(\mu)$ est probablement $(P=0,95)$ entre 9,02 et 13,53 larves d'Ephémères/relevé (aire $500 \mathrm{~cm}^{2}$ ). Ces limites peuvent être utilisées pour estimer que la population d'une section de ruisseau de $30 \mathrm{~m}^{2}$ est comprise entre 5412 et 8118 larves.

Table III. - Valeurs du $t$ de Student pour $95 \%$ des limites de confiance et pour les seuils de signification $5 \%$ dans les tests $t(v=$ nombre de degrés de liberté).

\begin{tabular}{rrrrrr}
\hline$v$ & $t$ & $v$ & $t$ & $v$ & $t$ \\
\hline 3 & 3.18 & 11 & 2.20 & 19 & 2.09 \\
4 & 2.78 & 12 & 2.18 & 20 & 2.09 \\
5 & 2.57 & 13 & 2.16 & 21 & 2.08 \\
6 & 2.45 & 14 & 2.14 & 22 & 2.07 \\
7 & 2.36 & 15 & 2.13 & 23 & 2.07 \\
8 & 2.31 & 16 & 2.12 & $24-26$ & 2.06 \\
9 & 2.26 & 17 & 2.11 & $27-28$ & 2.05 \\
10 & 2.23 & 18 & 2.10 & $29-30$ & 2.04 \\
\hline
\end{tabular}

Les tables des limites de confiance d'une variable de Poisson peuvent être utilisées quand $\mathrm{nm}$ est inférieur à 30 , ou quand les limites de conflance sont recherchées pour de simples nombres d'une série supposée appartenir à une loi de Poisson. La table 40 de Pearson et Hartley (1966) donne les $95 \%$ des limites de confiance dans la colonne intitulée $1-2 \alpha=0,95$ (ou $\alpha=0,025$ ), et c est soit le paramètre $m$ (estimé par $\bar{x}$ ), soit un simple nombre d'une loi de Poisson. Pour les valeurs de c supérieures à 300, il convient de consulter les plus grandes tables de Crow et Gardner (1959).

Quand les nombres d'une loi de Poisson sont utilisés pour estimer la moyenne de la population, la précision de l'estimation dépend de la taille du dénombrement, c'est-à-dire du nombre total d'invertébrés comptés; par exemple, les 95\% des limites de confiance sont $\pm 50 \%(8$ à 24$)$ pour un total de $16, \pm 20 \%(80$ à 120) pour un total de 100 , et $\pm 10 \%(360$ à 440$)$ pour un total de 400. Par suite, si la loi de Poisson est un modèle convenable, la précision de la moyenne estimée de la population est améliorée en comptant plus d'invertébrés. Ce résultat peut être obtenu en augmentant la dimension du relevé ou en combinant plusieurs petits relevés. 


\subsection{Limites de confiance de la moyenne d'un petit échantillon $(n<30)$ tiré d'une distribution contagieuse.}

La distribution spatiale d'une Population est souvent de type contagieux (§ 3.3.), avec une variance significativement plus grande que la moyenne. Le théorème central limite ne peut pas être appliqué aux petits échantillons tirés d'une distribution contagieuse. Il peut cependant être appliqué aux nombres quand ces derniers sont transformés, c'est-à-dire quand chaque nombre est remplacé par une fonction mathématique convenable (par exemple le logarithme). Une transformation correcte doit normaliser la distribution de probabilité des nombres, éliminer la dépendance de la variance par rapport à la moyenne, et assurer que les composantes de la variance sont additives (pour l'analyse de variance dans la comparaison des échantillons). Plusieurs transformations conviennent, qui sont dérivées des divers modèles mathématiques pour distribution contagieuse (voir Elliott 1971, chapitre 6). Pour les petits échantillons, la transformation la plus utile est probablement la transformation logarithmique, ou sa variante $\log (\mathrm{x}+$ 1). C'est une transformation approchée pour la plupart des petits éohantillons tirés d'une distribution contagieuse; elle est utilisée quand les autres transformations ne peuvent pas être appliquées, ou quand les échantillons sont trop petits pour justifier une transformation plus précise. Une transformation logarithmique : 1) est tacile à calculer et à appliquer, 2) satisfait habituellement la fonction la plus importante d'une transformation, — la stabilisation de la variance, 3) permet d'exprimer les limites de confiance en termes d'une moyenne dérivée, divisée et multipliée par un facteur commun. Une transformation logarithmique simple convient à la plupart des petits échantillons, mais $\log (x+1)$ doit être utilisé quand des valeurs zéro apparaissent dans l'échantillon.

Si les nombres $x_{1}, x_{2}, x_{3}, \ldots x_{n}$ sont transformés en $y_{1}, y_{2}, y_{3} \ldots$ $\mathbf{y}_{\mathbf{n}}(\mathbf{y}=\log \mathbf{x})$, la moyenne arithmétique des nombres transformés est $\mathrm{y}$ et la variance est $\mathrm{s}^{2}{ }_{y}$. Par suite, les $95 \%$ des limites de confiance pour $\overline{\mathrm{y}}$ sont : $\overline{\mathrm{y}} \pm \mathrm{t} \sqrt{\mathrm{s}_{\mathrm{y}}^{2} / \mathrm{n}}, \mathrm{t}$ étant trouvé dans la distribution du $t$ de Student (table III). Les antilogarithmes de ces limites donnent les $95 \%$ des limites de confiance pour la moyenne de la population. La moyenne dérivée (antilog $\bar{y}$ ) des nombres transformés est égale à la moyenne géométrique des nombres originaux; elle est toujours inférieure à la moyenne arithmétique $\overline{(x)}$ des nombres originaux.

Quand les limites de confiance sont écrites après la moyenne dérivée, elles doivent être données en antilogs, et le \pm de 
l'échelle logarithmique devient $\underset{\mathrm{y}}{\div}$ sur l'échelle arithmétique. Si $\overline{\mathrm{y}}=2,00$, l'erreur-standard $=0,15$ et $\mathrm{t}=2$, on aura $\mathrm{y} \pm \mathrm{t}$ (erreurstandard de $\bar{y})=2,00 \pm 0,30$ et les limites de confiance de la moyenne dérivée seront $100 \stackrel{\mathrm{x}}{\div} 2=50$ à 200 (et non $100 \pm 2$ ).

Un exemple de calcul des limiles de confiance est donné dans la figure 4 (exemple $4 \mathrm{~A}$ ). La moyenne dérivée (= moyenne

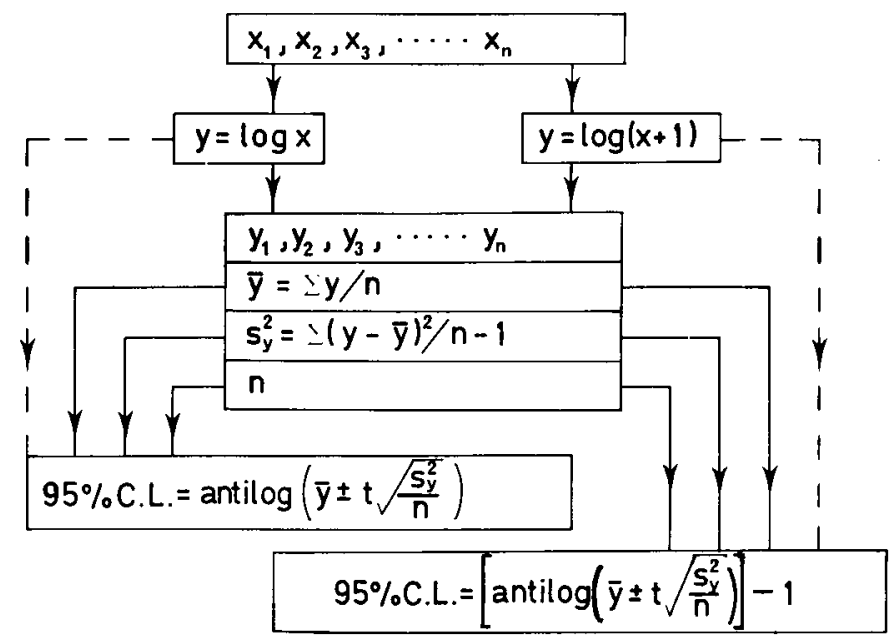

\begin{tabular}{|c|c|c|c|}
\hline \multicolumn{2}{|c|}{$4 \mathrm{~A}$} & & $4 B$ \\
\hline \multicolumn{2}{|c|}{$x=98,22,72,214,67$} & \multicolumn{2}{|c|}{$x=0,3,9,10$} \\
\hline \multicolumn{2}{|c|}{$y=1 \cdot 99,1 \cdot 34,1 \cdot 86,2 \cdot 33,1 \cdot 83$} & \multicolumn{2}{|c|}{$y=0 \cdot 00,0 \cdot 60,1 \cdot 00,1 \cdot 04$} \\
\hline \multicolumn{2}{|c|}{$\bar{y}=1 \cdot 870$} & \multicolumn{2}{|c|}{$\bar{y}=0.660$} \\
\hline \multicolumn{2}{|c|}{$s_{y}^{2}=0.127$} & \multicolumn{2}{|c|}{$s_{y}^{2}=0.233$} \\
\hline$n=5$ & $t=2 \cdot 78$ & $\mathrm{n}=4$ & $t=3.18$ \\
\hline \multicolumn{4}{|c|}{$y$} \\
\hline \multicolumn{4}{|c|}{$\begin{aligned} 95 \% \text { C.L. } & =\text { antilog }\left(1 \cdot 870 \pm 2 \cdot 78 \sqrt{\frac{0 \cdot 127}{5}}\right) \\
& =74 \cdot 13 \times 2 \cdot 77\end{aligned}$} \\
\hline & $95 \%$ C.L. & $\begin{array}{r}9(0.6 \\
\times 5.83\end{array}$ & $\left.18 \sqrt{\frac{0.233}{4}}\right]-$ \\
\hline
\end{tabular}

Fig. 4. - Calcul des $95 \%$ des limites de confiance (C. L.) de la moyenne d'un petit échantillon $(n<30)$ tiré d'une distribution contagieuse : échantillon dont les nombres ne comprennent pas de zéro (exemple $4 \mathrm{~A}$ ) et échantillon dont les nombres comprennent un zéro (exemple $4 \mathrm{~B}$ ). 
géométrique) $=74,13$ et les limites de confiance sont $74,13 \div$ $2,77=27$ à 205 . Par suite, les chances sont de 19 contre 1 pour que la moyenne vraie de la population $(\mu)$ soit entre 27 et 205 animaux par relevé. Ces limites peuvent ensuite être utilisées pour déterminer celles du nombre total d'animaux dans l'aire étudiée. Dans le deuxième exemple $(4 \mathrm{~B})$, un zéro apparaît et la transformation $\log (\mathrm{x}+1)$ est utilisée. On doit retrancher 1 des antilogs de la moyenne $(\bar{y})$ et les limites de confiance : $(4,57 \underset{\div}{\div} \overline{5}, 83)-1$, vont de 0 à 26 . Par suite, la moyenne vraie $(\mu)$ de la population est probablement $(\mathrm{P}=0,95)$ entre 0 et 26 individus par relevé. Ces limites sont très larges et ne peuvent être rapprochées qu'en augmentant le nombre de relevés $n$.

Comme la moyenne géométrique est toujours inférieure à la moyenne arithmétique, son emploi conduit à une sous-estimation de la moyenne arithmétique $(\mu)$ de la population. Il est donc plus exact d'utiliser la moyenne arithmétique $(x)$ de l'échantillon. Toutefois, il n'existe pas de méthode simple permettant d'estimer les limites de confiance de la moyenne arithmétique d'un petit échantillon tiré d'une distribution contagieuse. Un compromis possible est d'utiliser le facteur obtenu à partir de la transformation logarithmique et de l'appliquer à la moyenne arithmétique de l'échantillon.

Ce facteur est 2,77 dans l'exemple $4 \mathrm{~A}$, la moyenne arithmétique $(\bar{x})$ est 94,60 et les $95 \%$ des limites de confiance sont donc $94,60 \stackrel{\times}{\div} 2,77=34$ à 262. Par suite, il y a 19 chances contre 1 pour que la vraie moyenne $(\mu)$ de la population soit comprise entre 34 et 262 animaux par relevé. Ces valeurs sont un peu supérieures à celles calculées pour la moyenne géométrique $(27$ à 205) mais il y a une forte probabilité pour que ces deux intervalles de confiance comprennent la vraie moyenne $(\mu)$ de la population.

Il existe d'autres méthodes pour calculer les limites de confiance de la moyenne d'un petit échantillon tiré d'une distribution contagieuse, mais ces méthodes sont plus complexes et requièrent plusieurs hypothèses. Ce problème devrait trouver une solution dans un proche avenir.

\section{COMPARAISON DES ECHANTILLONS}

Deux échantillons ont rarement la même moyenne, et plusieurs échantillons aux moyennes différentes peuvent tous provenir de la même population. Un test statistique doit donc indiquer si la différence entre les moyennes de deux échantillons est significa- 
tive ou simplement compatible avec la fluctuation (ou «l'erreur») de la moyenne estimée de la population.

Il est d'abord procédé à «l'hypothèse nulle» $\left(\mathbf{H}_{0}\right)$. Il s'agit d'une simple description de ce qui est prévu selon une hypothèse standard, fixée à l'avance. Habituellement, l'hypothèse nulle suppose que les échantillons sont tirés de la même population et que, par suite, leurs moyennes fluctuent dans les limites de l'erreur acceptée pour la moyenne de la population. Pour comparer les moyennes de deux échantillons, il faut alors déterminer la probabilité (P) d'obtenir une différence semblable (ou plus grande) à celle existant entre ces moyennes lorsque les échantillons viennent de la même population (c'est-à-dire quand $\mathrm{H}_{\circ}$ est vraie). L'hypothèse nulle est acceptée quand cette probabilité (P) est grande, mais rejetée quand $\mathbf{P}$ est significativement petit. Un seuil de signification de $5 \%$ est habituellement utilisé $(P=0,05)$. $H_{\text {o }}$ est acceptée quand $P$ est plus grand que 0,05. Inversement, $H_{\circ}$ est rejetée quand $\mathrm{P}$ est inférieur à $0,05(\mathrm{P}<0,05)$, et les moyennes des échantillons sont jugées significativement différentes au seuil $5 \%$. Quand $P=0,05$, il n'y a qu'une chance sur vingt pour que la différence entre les moyennes (ou une différence plus grande) puisse être apparue par hasard sous $\mathrm{H}_{0}$. Un seuil de signification plus rigoureux peut être exigé pour le rejet de $\mathbf{H}_{\text {o }}$, par exemple une chance sur cent $(P=0,01)$, ou une chance sur mille $(P=0,001)$. Les différents seuils de signification sont souvent indiqués par des astérisques :

$$
\begin{aligned}
* & =\mathrm{P}<0,05 & & \text { significatif } \\
* * & =\mathrm{P}<0,01 & & \text { hautement significatif } \\
* * & =\mathrm{P}<0,001 & & \text { très hautement significatif. }
\end{aligned}
$$

Le seuil de $5 \%(\mathrm{P}=0,05)$ suffit dans la plupart des cas, mais il est toujours préférable d'indiquer la valeur de $P$ obtenue dans le test que de mentionner uniquement : $\mathbf{P}<0,05$.

Un test de signification fournit donc une base rationnelle de décision. Nous avons traité de différences entre moyennes d'échantillons, mais le même principe s'applique à la comparaison d'autres paramètres, par exemple la variance. Deux risques d'erreur accompagnent un test statistique. Le risque d'espèce I est de rejeter $H_{\circ}$ quand elle est vraie. Ce risque diminue avec le seuil de signification, par exemple le risque d'espèce $I$ est plus grand à $\mathrm{P}=\mathbf{0 , 0 5}$ qu'à $\mathrm{P}=\mathbf{0 , 0 1}$. Le risque d'espèce II est d'accepter $H_{\circ}$ quand elle est fausse. Pour une dimension d'échantillon donnée, l'abaissement du risque d'espèce I augmente la probabilité du risque d'espèce II. Une plus grande dimension 
d'échantillon, - c'est-à-dire un plus grand nombre de relevés —, réduit à la fois les deux espèces de risque. La puissance d'un test est définie par la probabilité de rejeter l'hypothèse $H_{o}$ quand elle est fausse; autrement dit, la puissance est : 1 - risque d'espèce I. Dans le test idéal, il est peu probable de rejeter l'hypothèse $\mathrm{H}_{\mathrm{o}}$ quand elle est vraie, et très probable de la rejeter quand elle est fausse. Par suite, un test statistique n'est pas un guide infaillible et ne peut jamais prouver une hypothèse donnée. Il existe toujours un risque d'erreur dans la décision du rejet ou du maintien de l'hypothèse $\mathrm{H}_{0}$.

Les tests de signification peuvent être "paramétriques» ou «non-paramétriques». Dans les tests paramétriques (5.1.) l'hypothèse nulle suppose qu'une distribution connue fournit un modèle convenable pour les échantillons (par exemple distribution normale, ou loi de Poisson) et spécifie que les paramètres de cette distribution (par exemple $\mu, \sigma^{2}, \lambda$ ) sont les mêmes que les échantillons étudiés. Les tests non-paramétriques (5.2.) n'impliquent pas cette hypothèse.

\subsection{Tests paramétriques.}

Les tests paramétriques suivants peuvent être utilisés quand la distribution de la population parente est connue par des expériences antérieures, par des considérations théoriques (par exemple le théorème central limite, ou lorsqu'elle est déduite de l'échantillon lui-même (par exemple loi de Poisson). Quand elles existent, les conditions d'utilisation du test doivent être énoncées, par exemple : «à condition que les deux échantillons viennent de la même population normalement distribuée, il existe une différence significative entre les moyennes des échantillons ». Quand il est évident que ces conditions n'existent pas, un test paramétrique ne doit pas être appliqué directement aux échantillons.

Les méthodes associées à la distribution normale (par exemple test $t$, analyse de variance, coefficient de corrélation) sont des méthodes paramétriques. Leur utilisation suppose au moins trois conditions : les données doivent suivre une distribution normale (ou une distribution normale à deux variables pour le coefficient de corrélation), la variance de l'échantillon doit être indépendante de la moyenne, et les composantes de la variance doivent être additives (pour l'analyse de variance). La distribution normale et les méthodes qui lui sont associées formant la majeure partie de nombreux manuels de statistiques, nous ne présentons ici qu'un guide succinct. Quand les conditions d'utilisation des méthodes paramétriques ne sont pas satisfaites, les nombres doivent être transformés (par exemple en remplaçant chaque nombre 
par son logarithme), et ces nombres transformés doivent satisfaire aux conditions précitées. Un autre procédé est l'application de méthodes non-paramétriques $(\S 5.2$.) aux données d'origine.

Le test de l'écart normal réduit peut être utilisé pour comparer les moyennes de deux grands échantillons $(n>50)$ ainsi que les moyennes de deux petits échantillons tirés d'une loi de Poisson (distribution au hasard). Pour ce test, la transformation des nombres n'est pas nécessaire, mais elle l'est généralement pour l'analyse de variance et les coefficients de corrélation. Avec les échantillons tirés d'une loi de Poisson, chaque nombre doit être remplacé par sa racine carrée (ou $\sqrt{\mathrm{x}+0,5}$ si certains nombres $<10$ ). Plusieurs transformations conviennent aux échantillons tirés de distributions contagieuses (voir Elliott 1971, chapitre 7); la transformation logarithmique est probablement la plus utilisée. La transformation des nombres est aussi nécessaire avant l'application d'un test $t$ aux petits échantillons $(n<50)$ tirés d'une distribution contagieuse. Le test de l'écart-réduit et le test $t$ sont décrits ci-dessous. Le lecteur est prié de se reporter aux manuels de statistique (par exemple Bailcy 1959, Campbell 1967, Snedecor et Cochran 1967, Elliott 1971) pour l'analyse de variance, le coefficient de corrélation et les analyses de régression.

\subsubsection{CoMparaison DES MOYENNES DE DEUX GRANDS Échantillons} (n $>50)$ : TEST DE L'ÉCART NORMAL RÉDUIT.

L'hypothèse nulle $\left(H_{o}\right)$ est que les deux échantillons viennent de la même population et par suite ont des moyennes et des variances égales $\left(\mu_{1}=\mu_{2}\right.$ et $\left.\sigma^{2_{1}}=\sigma^{2}{ }_{2}\right)$. La moyenne arithmétique $(\bar{x})$, la variance $\left(s^{2}\right)$ et le nombre de relevés $(n)$ sont $\bar{x}_{11} s^{2}{ }_{1} n_{1}$ pour le premier échantillon, $\overline{x_{2}} \quad s^{2}{ }_{2} \quad n_{2}$ pour le deuxième échantillon. Ces échantillons étant grands $(n>50)$, le théorème central limite est applicable. Les distributions parentes de $\bar{x}_{1}$ et de $\vec{x}_{2}$ sont donc supposées approximativement normales, et les erreurs-standard sont $\sqrt{\mathbf{s}_{1}{ }_{1} / \mathbf{n}_{1}}$ pour $\bar{x}_{1}, \sqrt{\mathbf{s}_{2}{ }_{2} / \mathbf{n}_{2}}$ pour $\bar{x}_{2}$. Dans l'hypothèse nulle, la différence des moyennes est égale à zéro $\left(\mu_{1}-\mu_{2}=0\right)$. Le théorème central limite s'appliquant à chaque échantillon, la différence des moyennes observées présente une distribution théoriquement normale autour de zéro, avec un écart-type égal à l'écart-type de la différence des moyennes. Par suite, la différence réelle $\left.\overline{(x}_{1}-\bar{x}_{2}\right)$ des moyennes des échantillons est « $d$ » écarts-types à partir de zéro où $\mathbf{d}$ est «l'écart-normal-réduit », c'est-à-dire une variable 
normale dont la moyenne est zéro et l'écart-type est l'unité. La valeur de $d$ est donnée par

$$
\mathrm{d}=\frac{\text { différence des moyennes des échantillons }}{\text { écart-type de la différence }} \text { (voir fig. } 5 \text { ). }
$$

Le rapport $d$ est négatif ou positif, et significatif au seuil $5 \%$ $(\mathrm{P}=0,05)$ quand sa valeur absolue est supérieure à 1,96 . La différence des moyennes des échantillons est significative au seuil $1 \%(\mathrm{P}=0,01)$ quand $\mathrm{d}>2,58$, significative au seuil $0,1 \%$ $(\mathrm{P}=0,001)$ quand $\mathrm{d}>3,29$. Cette méthode peut être appliquée à de grands échantillons $(\mathrm{n}>50)$ appartenant à des distributions au hasard, régulière ou contagieuse.

Dans l'exemple $5 \mathrm{~A},|\mathrm{~d}|$ est supérieur à 3,29 . La différence des moyennes des échantillons est donc très hautement significative $(\mathrm{P}<0,001)$, et $\mathrm{H}_{\mathrm{o}}$ est rejetée aux seuils $5 \%, 1 \%$ et $0,1 \%$.

La variance de la population étant estimée à partir du même échantillon que la moyenne ( $\sigma^{2}$ estimé par $\mathrm{s}^{2}$ ), il est aussi correct d'utiliser $t$ au lieu de $d$ dans la formule ci-dessus et de se référer aux tables de distribution du $t$ de Student (voir $\S$ 5.1.3.). Avec de grands échantillons, les valeurs de $d$ et de $t$ diffèrent peu, par exemple au seuil de signification $5 \%, d=1,96$ et $t=1,98$ avec 98 degrés de liberté( pour $\left.n_{1}=n_{2}=50\right)$.

\subsubsection{Comparaison des MOYenNes de DEUX PETtTS Échantillons (n< 50) TIRÉS D'UNE LOI de Poisson (DISTRIbution AU HASARD).}

L'écart normal réduit (d) est également utilisé pour comparer les moyennes de deux échantillons tirés d'une loi de Poisson quand le produit $\mathrm{nm}$ est supérieur à 30 pour chaque échantillon $\left(\mathrm{m}=\overline{\mathrm{x}}=\mathrm{s}^{2}\right.$ est l'estimation du paramètre $\lambda$ de Poisson). $\vec{x}$ est la meilleure estimation de $m$ et de $s^{2}$ dans la loi de Poisson (voir $\S 3.1$.) et remplace donc $\mathrm{s}^{2}$ dans la formule permettant d'obtenir $\mathrm{d}$ (voir fig. $5 \mathrm{~B}$ ).

L'estimation (m) du paramètre $\lambda$ de Poisson, la moyenne arithmétique $(\bar{x})$, et le nombre de relevés (n) sont $m_{1} x_{1} n_{1}$ pour le premier échantillon, $m_{2} \bar{x}_{2} n_{2}$ pour le deuxième échantillon. Chacun des produits $n_{1} m_{1}$ et $n_{2} m_{2}$ doit être supérieur à 30 .

Dans l'hypothèse nulle $\left(H_{0}\right)$, chaque échantillon vient de la même population et, par suite, les deux paramètres de Poisson sont égaux $\left(\lambda_{1}=\lambda_{2}\right)$. La valeur absolue de $d$ étant inférieure à 1,96 dans l'exemple $5 \mathrm{~B}$, la différence des moyennes des échantillons n'est pas significative au seuil de $5 \%(\mathrm{P}>0,05)$. L'hypothèse 
nulle $\left(H_{0}\right)$ est donc acceptée au seuil $5 \%$ et les deux paramètres de Poisson considérés comme égaux $\left(\lambda_{1}=\lambda_{2}\right)$; autrement dit, les moyennes ne sont pas significativement différentes.

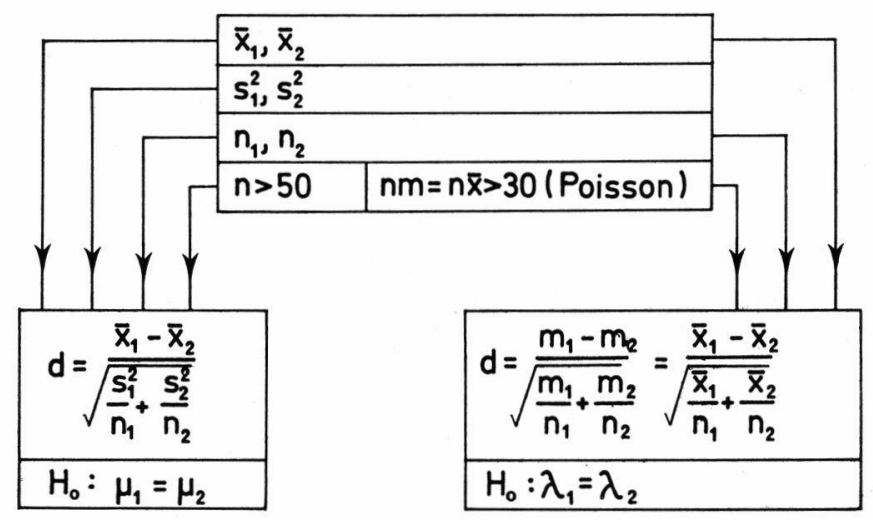

5A

\begin{tabular}{l}
$\bar{x}_{1}=10.125, \bar{x}_{2}=12.245$ \\
\hline$s_{1}^{2}=7.465, s_{2}^{2}=8.855$ \\
\hline$n_{1}=80, \quad n_{2}=60$ \\
$\downarrow$ \\
$d=\frac{10.125-12.245}{\sqrt{\frac{7.465}{80}+\frac{8.855}{60}}}$ \\
$=-4.32(P<0.001)$
\end{tabular}

$5 B$

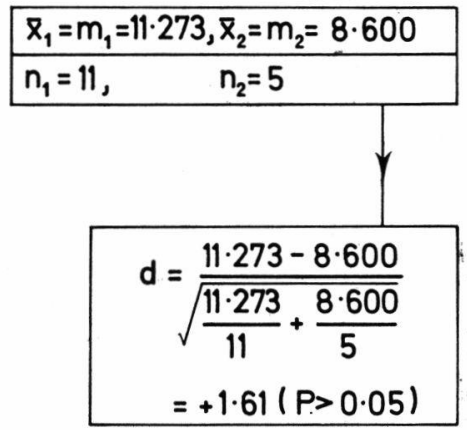

Fig. 5. - Comparaison des moyennes de deux grands échantilons (n $>50$ dans l'exemple $5 \mathrm{~A}$ ) et des moyennes de deux petits échantillons tirés d'une loi de Poisson (distribution au hasard) avec le produit $\mathrm{nm}>30$ (exemple 5 B).

La table 36 de Pearson et Hartley (1966) est utilisée quand nm est inférieur à 30 pour un seul ou pour les deux échantillons, ou quand les comparaisons portent sur de simples nombres appartenant à une loi de Poisson. Les deux variables de Poisson sont nommées a et $\mathbf{b}(\mathbf{b}<\mathbf{a})$, et la somme des deux nombres $(r=a+b)$ est donnée par la colonne de gauche de la table 36 . b est significativement inférieur à a quand, pour un seuil donné de probabilité, b est inférieur ou égal à la valeur de la table (test unilatéral ou single-tail test). Les seuils de probabilité doivent être doublés pour 
un test portant sur la différence entre a et b (test bilatéral ou double-tail test); c'est-à-dire que la colonne intitulée 0,025 est utilisée pour un test au seuil de signification $5 \%$, et 0,005 au seuil de $1 \%$. Par exemple, 12 et 3 étant les moyennes de deux échantillons d'une série de Poisson $(\mathrm{nm}<30)$, on aura : $\mathrm{a}=12, \mathrm{~b}=3$ et $r=15$. Comme $b$ est égal à la valeur de la table pour $r=15$ et $\mathbf{P}=\mathbf{0 , 0 2 5}$, les moyennes sont significativement différentes au seuil $5 \%$, mais non au seuil $1 \%$ (valeur de la table $=2$ pour $\mathrm{r}=15$ et $\mathbf{P}=0,005)$.

5.1.3. Comparaison des MoyenNes de DEUX PETITS ÉCHantillons (n $<50$ ) TIRÉs D'UNE distribution CONTAgIEUSE : TEST F ET TEST $t$.

Le test $t$ est décrit dans les manuels de statistique (par exemple Bailey 1959, Snedecor et Cochran 1967). Nous en résumons les calculs dans la figure 6. L'hypothèse nulle $\left(H_{0}\right)$ est que les deux échantillons sont tirés de deux populations normalement distribuées, avec des moyennes et des variances égales $\left(\mu_{1}=\mu_{2}, \sigma^{2}{ }_{1}=\sigma^{2}{ }_{2}\right)$. La condition de base d'un test $t$ est que la distribution parente soit au moins approximativement normale, avec une variance indépendante de la moyenne. Le test $t$ ne peut donc pas être appliqué directement aux petits échantillons appartenant à des distributions contagieuses : une transformation des nombres est nécessaire. La transformation la plus utile pour les petits échantillons est probablement la transformation logarithmique (voir $\S 4.3$.), et un exemple de son utilisation pour un test $t$ est présenté dans la figure 6 .

Dans un test $t$, la différence entre les moyennes des échantillons est significative au seuil $5 \%(P=0,05)$ quand la valeur calculée de $t$ est supérieure à celle de la table pour $v=n_{1}+n_{2}-2$ degrés de liberté (voir table III). Les seuils de signification de $1 \%$ ( $\mathrm{P}=\mathbf{0 , 0 1})$ et de $0,1 \%(\mathrm{P}=\mathbf{0 , 0 0 1})$ sont habituellement donnés dans les tables de distribution de $t$ (par exemple table 12 de Pearson et Hartley 1966).

Une différence significative entre les variances entraîne également une valeur significative de $t$. Par suite, le test $t$ est souvent précédé par un test $F$ pour juger de l'égalité des deux variances estimées $\mathbf{s}^{2}$ et $\mathrm{s}^{2}{ }_{2}(f i g .6)$, avec $\mathrm{s}^{2}{ }_{1}$ toujours supérieure à $\mathbf{s}^{2}{ }_{2}$. L'hypothèse nulle $\left(H_{0}\right)$ est que les variances des populations sont égales $\left(\sigma^{2}{ }_{1}=\sigma^{2}{ }_{2}\right)$. La différence entre les variances des échantillons est significative quand la valeur calculée de $F$ est supérieure à la valeur de la table pour $v_{1}=n_{1}-1$ degrés de liberté au numérateur et $v_{2}=n_{2}-1$ degrés de liberté au dénominateur. Un test sur les différences (ou test bilatéral) étant ici nécessaire, la table des valeurs supérieures à $2,5 \%$ est utilisée pour le seuil de signi- 
fication $5 \%(\mathrm{P}=0,05)$, et la table des valeurs supérieures à $0,5 \%$ pour le seuil de signification $1 \%(\mathrm{P}=0,01)$ (voir tables des pourcentages de distribution de $\mathrm{F}$, par exemple Pearson et
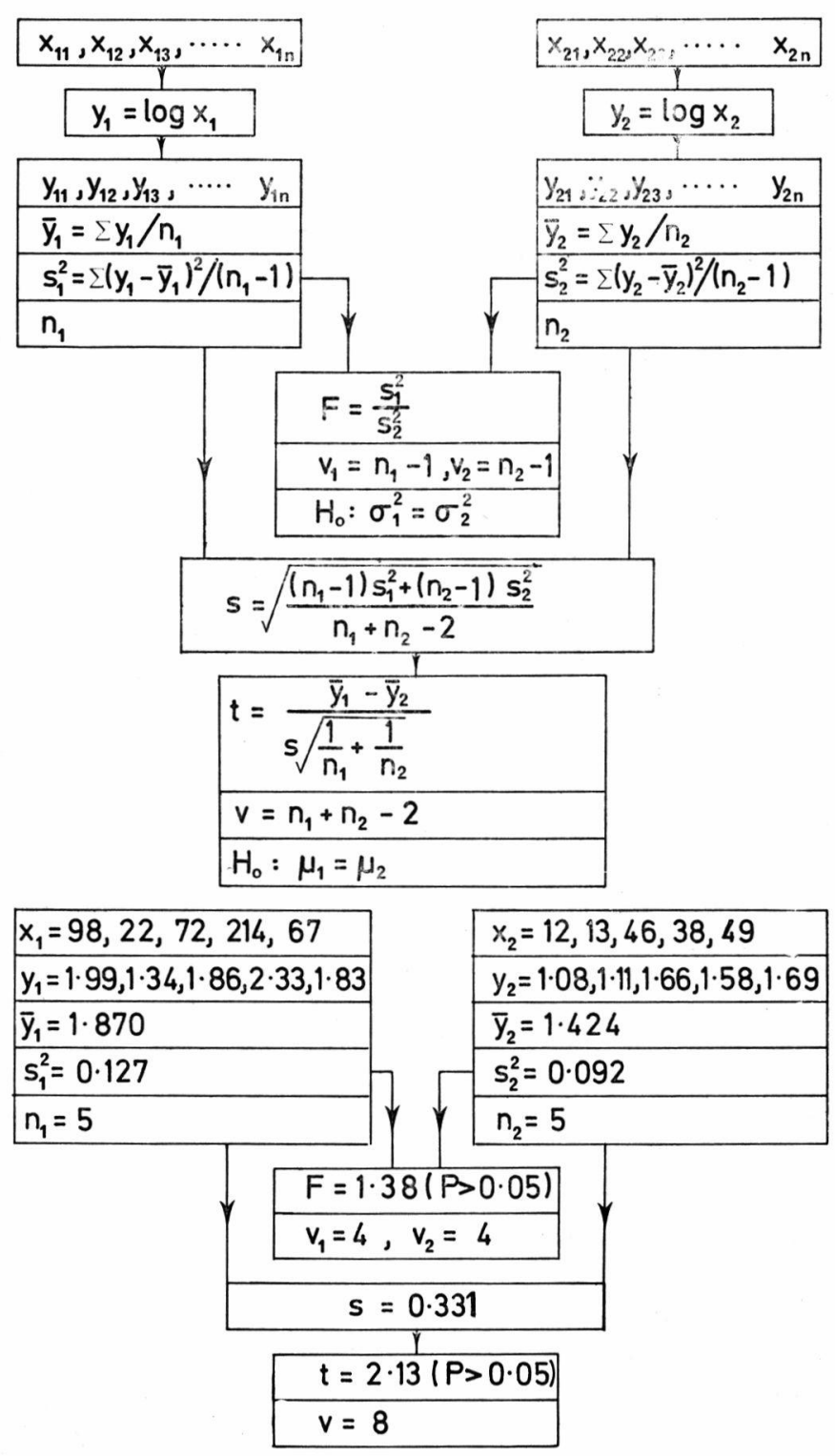

Fig. 6. - Comparaison des moyennes de deux petits échantillons $(n<50)$ tirés d'une distribution contagieuse. Les variances sont d'abord comparées par le test $F$, puis les moyennes par le test $t$. 
Hartley 1966, table 18). L'égalité des variances est une condition nécessaire de l'hypothèse nulle selon laquelle les deux échantillons sont tirés de la même population normalement distribuée. $H_{o}$ est ici rejetée quand une différence significative apparaît entre les moyennes ou les variances.

Dans l'exemple de la figure 6 , la valeur calculée $F=1,38$ est bien en dessous de la valeur 9,60 de la table pour $\mathrm{v}_{1}=4$ et $\mathrm{v}_{2}=4$ degrés de liberté (valeurs supérieures à $2,5 \%$ de la table 18 , Pearson et Hartley 1966). Par suite, les variances des deux échantillons ne sont pas significativement différentes $(P>0,05)$, et $H_{\circ}$ n'est pas contredite. La valeur calculée $t=2,13$ est aussi en dessous de la valeur 2,31 de la table pour $\mathrm{v}=\mathbf{8}$ degrés de liberté (table III). Par suite, les moyennes des deux échantillons ne sont pas significativement différentes $(P>0,05)$, et $H_{0}$ n'est pas contredite. L'hypothèse nulle $\left(H_{o}\right)$ peut alors être acceptée, mais son non-rejet peut toujours être dû à de petits échantillons et des données très variables. Un deuxième exemple peut être essayé par le lecteur. Les nombre sont $4,5,8,14,14,15,15,19,28,36$ pour l'échantillon 1 ; et $2,4,5,7,12$ pour l'échantillon 2 . Après transformation logarithmique de ces nombre : $\overline{\mathbf{y}}_{1}=1,115 ; \mathrm{s}^{2}{ }_{1}=0,092$; $\bar{y}_{2}=0,706 ; \mathrm{s}^{2}{ }_{2}=0,084 . \mathrm{F}=1,10(\mathrm{P}>0,05)$ et $\mathrm{F}$ de la table $=8,90$ pour $\mathrm{v}_{1}=9$ et $\mathrm{v}_{2}=4$. Les variances ne sont donc pas significativement différentes $(P>0,05) . t=2,49$ alors que $t$ de la table $=2,16$ pour $\mathrm{v}=13$ degrés de liberté. La valeur calculée étant supérieure à celle de la table, la différence des moyennes des échantillons est significative au seuil $5 \%(\mathrm{P}<0,05)$, et $\mathbf{H}_{\circ}$ est rejetée. Noter que la valeur calculée de $t$ est inférieure à celle de la table $(3,01)$ pour $P=0,01$ : les moyennes ne sont donc pas significativement différentes au seuil $1 \%(\mathrm{P}>0,01)$. La différence entre les moyennes des échantillons est donc significative mais ne peul pas être considérée comme hautement significative.

\subsection{Méthodes non-paramétriques.}

Dans les tests paramétriques (par exemple test $t$ ), l'hypothèse nulle $\left(\mathbf{H}_{0}\right)$ suppose une distribution parente particulière (par exemple normale) avec des paramètres (par exemple moyenne $\mu$, variance $\sigma^{2}$ ) semblables à ceux des échantillons. Il n'en est rien pour les méthodes non-paramétriques, qui peuvent s'appliquer aux échantillons quand les conditions requises pour les méthodes «normales»ne sont pas satisfaites. Par suite, les tests non-paramétriques remplacent les méthodes «normales» telles qu'elles étaient appliquées sur nombres transformés; ils conviennent particulièrement aux petits échantillons de distributions contagieuses. 
D'un point de vue pratique, les calculs des tests non-paramétriques sont souvent simples, alors que ceux des «tests normaux» peuvent être laborieux, notamment quand les données sont transformées.

Les tests non-paramétriques sont parfois critiqués en ce qu'ils n'utilisent pas toute l'information fournie par l'échantillon. Quand les suppositions d'un test paramétrique sont valides, ce test est plus puissant que tout autre pour rejeter $\mathbf{H}_{0}$ quand $\mathbf{H}_{0}$ est fausse. Mais beaucoup de tests non-paramétriques sont presque aussi efficaces que leurs équivalents paramétriques, dans les conditions mêmes de validité de test paramétrique. Quand ces conditions ne sont pas remplies, le test non-paramétrique est habituellement plus puissant que son équivalent paramétrique.

Le test $U$ de Mann-Whitney pour comparer deux échantillons, équivalent non-paramétrique du test $t$, est inclus dans ce guide. Quelques méthodes associées à la distribution de $\chi^{2}$ sont aussi décrites. Le lecteur est renvoyé à d'autres exposés (par exemple Siegel 1956, Elliott 1971) pour les équivalents non-paramétriques de l'analyse de variance (par exemple test de Quenouille, analyse par rangs simple de Kruskal-Wallis, analyse par rangs en blocs casualisés de Friedman) et des coefficients de corrélation (par exemple coefficient de corrélation de rang de Spearman).

\subsubsection{Comparaison de DEUX Échantillons : test U de ManN- WHITNEY.}

L'efficacité du test $\mathrm{U}$, jamais inférieure à $86 \%$, est comprise entre $90 \%$ et $96 \%$ pour des données normales; elle peut être beaucoup plus élevée que l'efficacité des méthodes «normales» lorsqu'il s'agit de données non-normales (Siegel 1956, Wilcoxon et Wilcox 1964).

L'hypothèse nulle $\left(\mathrm{H}_{\circ}\right)$ est que deux échantillons indépendants, pris au hasard, sont tirés de populations ayant la même distribution parente et les mêmes médianes (valeurs médianes). Dans ce cas, $H_{0}$ ne spécifie pas la forme de la distribution parente, mais suppose seulement qu'elle est la même pour les deux échantillons. $\mathrm{H}_{\mathrm{o}}$ est vérifiée par le test de $\mathrm{U}$ de Mann-Whitney, qui est un test d'ordre de rang, c'est-à-dire que les valeurs dénombrées (ou nombres) sont remplacées par leur rang dans une séquence unique.

La marche à suivre est la suivante :

1. Considérer ensemble les valeurs des deux échantillons et disposer ces valeurs sur un seul rang du plus petit au plus grand. $\mathrm{n}_{1}=$ nombre de valeurs de l'échantillon $1, \mathrm{n}_{2}=$ nombre de valeurs de l'échantillon 2.

2. Affecter un rang $r$ à chaque valeur. Les rangs se disposent de 1 pour la plus faible valeur à $N$ pour la plus élevée $\left(N=n_{1}+n_{2}\right)$. 


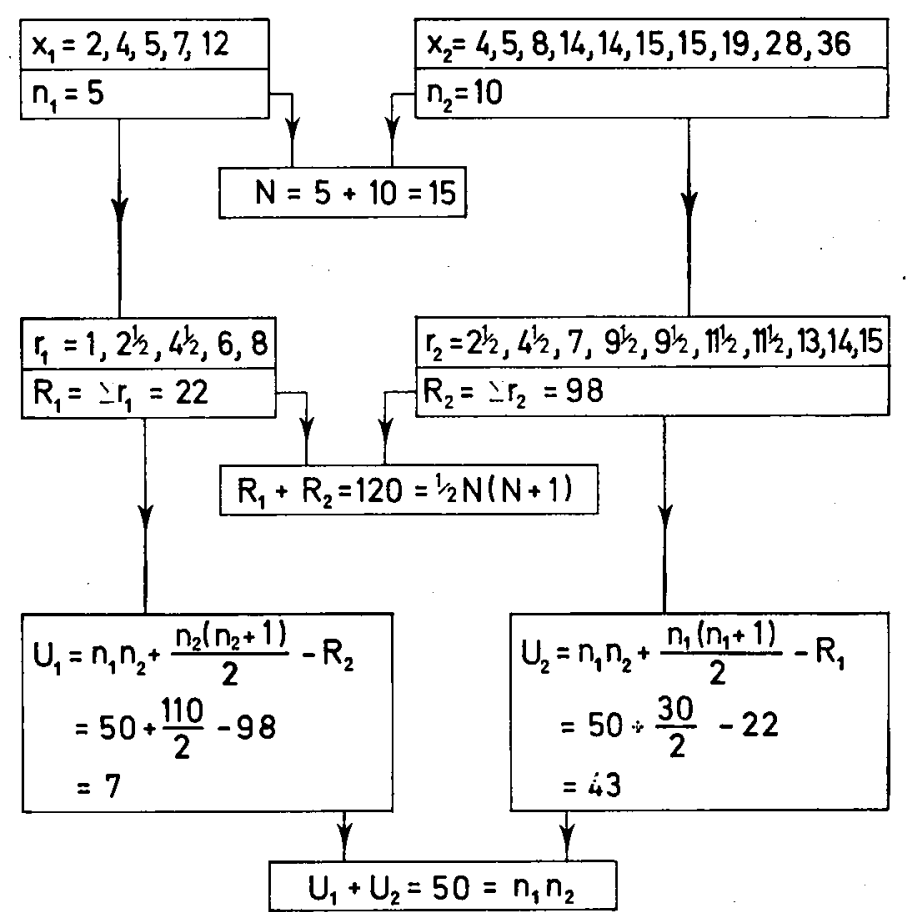

Fig. 7. - Comparajson de deux échantillons : test $U$ de Mann-Whitney.

Les valeurs égales sont affectées de la moyenne des rangs communs.

3. Totaliser les rangs pour chaque échantillon. $R_{1}=$ somme des rangs de l'échantillon $1, R_{2}=$ somme des rangs de l'échantillon 2. Vérifier que $R_{1}+R_{2}=\frac{1}{2} N(N+1)$.

4. Calculer les paramètres $U_{1}$ et $U_{2}$ (formules dans la figure 7 ) et vérifier que $U_{1}+U_{2}=n_{1} n_{2}$. Comparer la plus petite des deux valeurs $U_{1}$ et $U_{2}$ à la valeur convenable de $U$ de la table IV (précisée par les valeurs $n_{1}$ et $n_{2}$ ). Si la valeur calculée de $U$ est égale ou inférieure à la valeur de la table, $H_{0}$ est rejetée au seuil de signification $5 \%(P=0,05)$. On notera que les petites valeurs de $\mathrm{U}$ conduisent à un rejet.

5. La table IV ne peut pas être utilisée quand $n_{1}$ ou $n_{2}$ est supérieur à 20. Lorsque $n_{1}$ et $n_{2}$ augmentent, la distribution de $U$ tend rapidement vers une distribution de fréquence normale, avec pour moyenne $: \frac{n_{1} n_{2}}{2}$. Par suite, calculer l'écart normal réduit 
d :

$$
d=\frac{U-\left(n_{1} n_{2}\right) / 2}{\frac{\sqrt{n_{1} n_{2}(N+1)}}{12}}
$$

$\mathbf{H}_{0}$ est rejetée au seuil $5 \%(\mathrm{P}=0,05)$ quand la valeur absolue de $\mathrm{d}$ est supérieure à 1,96 au seuil $1 \%(\mathrm{P}=0,01)$ quand $\mathrm{d}>2,58$ et au seuil 0,1\% $(\mathrm{P}=0,001)$ quand $\mathrm{d}>3,29$.

Dans l'exemple (fig. 7), la plus petite des deux valeurs de U est $U_{1}=7$. Cette valeur calculée étant inférieure à la valeur $U=8$ de la table pour $n_{1}=5$ et $n_{2}=10$ (voir table IV), $H_{v}$ est rejetée au seuil de signification $5 \%(P<0,05)$. Il s'ensuit que la médiane de l'échantillon 2 est significativement plus élevée que celle de l'échantillon $1(P<0,05)$. La valeur calculée de $U$ est juste en dessous de la valeur de la table au seuil $5 \%$. Des tables plus détaillées (par exemple Siegel 1956, table $\mathrm{k}$ ) révéleraient que $\mathrm{U}$ n'est pas significatif au seuil $2 \%(\mathrm{P}>0,02)$. Cet exemple est identique à celui proposé au $\S 5.1 .3$. Par suite, le test $U$ se révèle ici aussi sensible que le test $t$ qui indiquait une différence significative entre les moyennes au seuil $5 \%$, mais non au seuil $1 \%$.

TABLE IV. - Valeurs du paramètre $U$ au seuil de signification $5 \%$. $n_{1}$ et $n_{z}$ indiquent les dénombrements réalisés pour chaque échantillon. Noter que les

\begin{tabular}{|c|c|c|c|c|c|c|c|c|c|c|c|c|c|c|c|c|c|c|c|}
\hline$n_{1} \mid n_{r}$ & 2 & 3 & 4 & 5 & 6 & 7 & 8 & 9 & 10 & 11 & 12 & 13 & 14 & 15 & 16 & 17 & 18 & 19 & 20 \\
\hline 2 & & & & & & & 0 & 0 & 0 & 0 & 1 & 1 & 1 & 1 & 1 & 2 & 2 & 2 & 2 \\
\hline$\overline{3}$ & & & & 0 & 1 & 1 & 2 & 2 & 3 & 3 & 4 & 4 & 5 & 5 & 6 & 6 & 7 & 7 & 8 \\
\hline 4 & & & 0 & 1 & 2 & 3 & $\overline{4}$ & 4 & 5 & 6 & 7 & 8 & 9 & 10 & 11 & 11 & 12 & 13 & 13 \\
\hline 5 & & 0 & 1 & 2 & 3 & 5 & 6 & 7 & 8 & 9 & 11 & 12 & 13 & 14 & 15 & 17 & 18 & 19 & 20 \\
\hline 6 & & 1 & 2 & 3 & 5 & 6 & 8 & 10 & 11 & 13 & 14 & 16 & 17 & 19 & 21 & 22 & 24 & 25 & 27 \\
\hline 7 & & 1 & 3 & 5 & 6 & 8 & 10 & 12 & 14 & 16 & 18 & 20 & 22 & 24 & 26 & 28 & 30 & 32 & 34 \\
\hline 8 & 0 & 2 & 4 & 6 & 8 & 10 & 13 & 15 & 17 & 19 & 22 & 24 & 26 & 29 & 31 & 34 & 36 & 38 & 41 \\
\hline 9 & 0 & 2 & 4 & 7 & 10 & 12 & 15 & 17 & 20 & 23 & 26 & 28 & 31 & 34 & 37 & 39 & 42 & 45 & 48 \\
\hline 10 & 0 & 3 & 5 & 8 & 11 & 14 & 17 & 20 & 23 & 26 & 29 & 33 & 36 & 39 & 42 & 45 & 48 & 52 & 55 \\
\hline 11 & 0 & 3 & 6 & 9 & 13 & 16 & 19 & 23 & 26 & 30 & 33 & 37 & 40 & 44 & 47 & 51 & 55 & 58 & 62 \\
\hline 12 & 1 & 4 & 7 & 11 & 14 & 18 & 22 & 26 & 29 & 33 & 37 & 41 & 45 & 49 & 53 & 57 & 61 & 65 & 69 \\
\hline 13 & 1 & 4 & 8 & 12 & 16. & 20 & 24 & 28 & 33 & 37 & 41 & 45 & 50 & 54 & 59 & 63 & 67 & 72 & 76 \\
\hline 14 & 1 & 5 & 9 & 13 & 17 & 22 & 26 & 31 & 36 & 40 & 45 & 50 & 55 & 59 & 64 & 67 & 74 & 78 & 83 \\
\hline 15 & 1 & 5 & 10 & 14 & 19 & 24 & 29 & 34 & 39 & 44 & 49 & 54 & 59 & 64 & 70 & 75 & 80 & 85 & 90 \\
\hline 16 & 1 & 6 & 11 & 15 & 21 & 26 & 31 & 37 & 42 & 47 & 53 & 59 & 64 & 70 & 75 & 81 & 86 & 92 & 98 \\
\hline 17 & 2 & 6 & 11 & 17 & 22 & 28 & 34 & 39 & 45 & 51 & 57 & 63 & 67 & 75 & 81 & 87 & 93 & 99 & 105 \\
\hline 18 & 2 & 7 & 12 & 18 & 24 & 30 & 36 & 42 & 48 & 55 & 61 & 67 & 74 & 80 & 86 & 93 & 99 & 106 & 112 \\
\hline 19 & 2 & 7 & 13 & 19 & 25 & 32 & 38 & 45 & 52 & 58 & 65 & 72 & 78 & 85 & 92 & 99 & 106 & 113 & 119 \\
\hline 20 & 2 & 8 & 13 & 20 & 27 & 34 & 41 & 48 & 55 & 62 & 69 & 76 & 83 & 90 & 98 & 105 & 112 & 119 & 127 \\
\hline
\end{tabular}
petites valeurs de $U$ causent le rejet de $H_{0}$ au seuil $5 \%$.

5.2.2. $\chi^{2}$ ET TABLES DE CONTINGENCE.

La distribution de $\chi^{2}$ a de nombreux usages en statistique. Son emploi pour tester la conformité de l'observation à l'hypothèse 
s'étend des tests d'ajustement à toute situation dans laquelle une hypothèse de base spécifie les proportions ou les probabilités d'une série d'observations appartenant à plusieurs groupes.

Un exemple courant, avec simplement deux groupes, est la distribution des sexes. On compte par exemple 53 mâles et 67 femelles dans une capture totale de 120 animaux. L'hypothèse nulle $\left(\mathrm{H}_{\mathrm{o}}\right)$ suppose l'égalité des sexes, c'est-à-dire 60 mâles et 60 femelles. Par suite :

$$
x^{2}=\Sigma \frac{(\text { observé }- \text { théorique })^{2}}{\text { théorique }}=\frac{(53-60)^{2}}{60}+\frac{(67-60)^{2}}{60}=1,63
$$

avec $\mathrm{v}=\mathrm{k}-1=2-1=1$ degré de liberté $(\mathrm{k}=$ nombre de groupes $)$. La valeur calculée de $x^{2}$ étant bien inférieure à la valeur 3,84 de la table $(v=1$ et $Q=0,050)$ de Pearson et Hartley (1966), $H_{o}$ n'est pas rejetée au seuil $5 \%(P>0,05)$. Il n'apparaît donc pas de différence significative par rapport à l'égalité supposée des sexes.

Cette méthode peut être étendue à $k$ groupes. Quand $k=2$, chaque valeur théorique doit être au moins égale à 5 . Quand $k>2$, $20 \%$ des fréquences théoriques peuvent être inférieures à 5, et aucune ne doit être inférieure à 1 (Cochran 1954). Il est souvent difficile d'estimer les valeurs théoriques de $\mathrm{H}_{0}$, aussi ce test n'at-il qu'une valeur limitée. De plus, l'hypothèse nulle $\left(H_{o}\right)$ habituelle est que toutes les valeurs théoriques sont les mêmes, ce qui est rarement réalisé. Cependant, cette méthode convient à certaines comparaisons.

Par exemple, les captures observées de 5 pièges $(k=5)$ sont 6 , $8,16,5,18 . H_{0}$ est que tous les pièges ont la même efficacité et par suite, que les captures théoriques sont les mêmes (captures théoriques $=$ captures totales $/ k=53 / 5=10,6)$. D'où :

$$
\chi^{2}=\frac{(6-10,6)^{2}}{10,6}+\ldots+\frac{(18-10,6)^{2}}{10,6}=\frac{143,2}{10,6}=13,5
$$

avec $\mathrm{v}=\mathrm{k}-1=4$ degrés de liberté. Cette valeur de $\chi^{2}$ étant bien supérieure à la valeur $9,49(\mathrm{v}=4$ et $\mathrm{Q}=0,050)$ de la table II, $\mathrm{H}_{0}$ est rejetée au seuil $5 \%(\mathrm{P}<0,05)$. Les 5 pièges n'ont donc pas la même efficacité.

Quand les individus sont classés selon deux critères, chaque classification comprenant deux ou plusieurs catégories, les données sont disposées dans une «table de contingence » avec $k$ colonnes et 1 lignes. Les individus de chaque case de la table appartiennent à la même catégorie dans chaque système de classification. L'hypothèse nulle $\left(\mathrm{H}_{\mathrm{o}}\right)$ est soit que les proportions de la colonne sont égales, soit que les proportions de la ligne sont égales. Les valeurs théoriques de $\mathrm{H}_{0}$ sont calculées pour chaque case de la table. Les 
valeurs observées et théoriques pour chaque case sont comparées par le test $\chi^{2}$ habituel, et le $\chi^{2}$ total pour l'ensemble de la table est comparé aux tables de $\chi^{2}$ appropriées, avec $\mathrm{v}=(\mathrm{k}-1)$ (l-1) degrés de liberté. Comme le paramètre $\chi^{2}$ est le même pour tester l'une ou l'autre des hypothèses nulles, on dispose là d'un bon test d'association entre les deux classifications. Par suite, le test $\chi^{2}$ est utilisé pour déceler toute différence entre lignes dans les proportions de colonne à colonne, ou toute différence entre colonnes dans les proportions de ligne à ligne.

La table la plus simple, utilisée pour comparer les proportions de deux échantilons indépendants, n'a que deux lignes et deux colonnes. Une table $2 \times 2$ peut aussi être utilisée pour tester l'association entre deux espèces (voir Bonnet 1964, Greig-Smith 1964, chap. 4). Des tables plus complexes ont $\mathrm{k}$ colonnes et $\mathrm{l}$ lignes. Lorsque $\mathbf{k}$ ou $\mathbf{l}$ est supérieur à 2 , moins de $20 \%$ des cases doivent avoir des valeurs théoriques inférieures à 5 , et aucune case ne doit avoir de valeur théorique inférieure à 1 (Cochran 1954). Si ces conditions ne sont pas remplies, les valeurs des cases adjacentes doivent être combinées. Lorsque la table de contingence est du type $2 \times 2$, les valeurs théoriques doivent être au moins égales à 5 . Si cette condition n'est pas remplie, il est évidemment impossible de combiner les valeurs adjacentes, et un test exact doit être utilisé (voir Bailey 1959, chap. 7; Pearson et Hartley 1966, section 19). De plus, la théorie du $\chi^{2}$ suppose que les nombres impliqués dans une table de contingence sont des variables continues et non discrètes. Par suite, la correction de Yates est utilisée pour une petite table de contingence.

L'utilisation d'une table $2 \times 2$ est illustrée par l'exemple suivant. Plusieurs échantillons de fond ont été prélevés dans un ruisseau pierreux, près et à distance de la rive. Des nombres égaux de relevés composent chaque groupe d'échantillons. Les larves d'une Ephémère ont été comptées dans chaque échantillon, les derniers stades étant comptés séparément des autres stades. Les valeurs figurent dans la table de contingence ci-dessous, avec les symboles usuels.

\begin{tabular}{|c|c|c|c|}
\hline Echantillons & $\begin{array}{l}\text { Dernier } \\
\text { stade }\end{array}$ & $\begin{array}{l}\text { Autres } \\
\text { stades }\end{array}$ & Totaux \\
\hline $\begin{array}{l}\text { près du bord } \\
- \text { loin du bord } \\
\text { totaux }\end{array}$ & $\begin{array}{r}a=42 \\
c=12 \\
a+c=54\end{array}$ & $\begin{array}{r}b=96 \\
d=104 \\
b+d=200\end{array}$ & $\begin{array}{r}\mathrm{a}+\mathrm{b}=\mathbf{1 3 8} \\
\mathrm{c}+\mathrm{d}=\mathbf{1 1 6} \\
\mathrm{n}=\mathrm{a}+\mathrm{b}+\mathrm{c}+\mathrm{d}=\mathbf{2 5 4}\end{array}$ \\
\hline
\end{tabular}


L'hypothèse nulle $\left(H_{o}\right)$ est que les proportions sont les mêmes dans les deux groupes d'échantillons, c'est-à-dire qu'il n'y a pas d'association entre les deux classifications. Le $\chi^{2}$ pourrait être calculé en déterminant d'abord les valeurs théoriques dans chacune des 4 cases, puis en comparant les valeurs observées et théoriques dans chaque case. Pour une table de contingence $2 \times 2$, il est plus facile d'utiliser la formule abrégée suivante :

$$
x^{2}=\frac{\mathrm{n}(/ \mathrm{ad}-\mathrm{bc} /-\mathrm{n} / 2)^{2}}{(\mathrm{a}+\mathrm{b})(\mathrm{c}+\mathrm{d})(\mathrm{a}+\mathrm{c})(\mathrm{b}+\mathrm{d})}
$$

où / ad - bc/ est la valeur absolue de (ad -- bc), et $n / 2$ la correction de Yates pour la continuité.

$$
\begin{aligned}
\chi^{2} & =\frac{254[/(42)(104)-(96)(12) /-127]^{2}}{(138)(116)(54)(200)} \\
& =\frac{2423647934}{172886400} \\
& =14,02 \text { avec } \mathrm{v}=(\mathrm{k}-1)(\mathrm{l}-1)=1 \text { degré de liberté. }
\end{aligned}
$$

Comme cette valeur de $\chi^{2}$ est supérieure à la valeur $5 \%$ de la table II, et supérieure à la valeur 10,83 pour $0,1 \%$ de la table 8 de Pearson et Hartley (1966), l'association entre les deux classifications est hautement significative $(P<0,001)$. Par suite, $H_{\circ}$ est rejetéc au seuil $0,1 \%$, et il est prouvé que la proportion des derniers stades est significativement plus élevée près du bord.

L'exemple suivant illustre l'emploi de plus grandes tables de contingence. Des échantillons du fond ont été prélevés en quatre stations d'un ruisseau, et les larves de Sericostoma personatum (Trichoptera) ont été comptées dans chaque échantillon. Six stades larvaires ont été distingués. Le total des captures de chaque stade à chaque station a été placé dans une table de contingence (table $\mathrm{V}$ ) à six colonnes $(\mathrm{k}=6)$ et quatre lignes $(\mathrm{l}=4)$. L'hypothèse nulle $\left(H_{o}\right)$ est que la proportion des larves de chaque stade est la même aux quatre stations, c'est-à-dire qu'il n'y a pas d'association entre les deux classifications. Les valeurs théoriques ont été calculées pour chaque case de la table : ces valeurs théoriques devraient apparaître si $H_{\circ}$ était correcte. Pour trouver les valeurs théoriques de chaque case, diviser le produit des totaux des lignes et colonnes correspondantes par le total général de l'ensemble de la table. Ainsi, pour la deuxième case de la deuxième ligne, captures observées $=12$, et captures théoriques $=$ 
$\frac{(\text { total de la ligne) (total de la colonne) }}{\text { total général }}=\frac{(148)(109)}{978}=16,50$.

Les valeurs observées et théoriques pour chaque case sont comparées par le test $\chi^{2}$ habituel, et le $\chi^{2}$ total pour l'ensemble de la table est :

$$
\begin{aligned}
\chi^{2} & =\Sigma \frac{(\text { observé }- \text { théorique })^{2}}{\text { théorique }}=\frac{(26-7,68)^{2}}{7,68}+\ldots+\frac{(82-59,97)^{2}}{59,97} \\
& =205,09 \text { avec } \mathrm{v}=(\mathrm{k}-1)(1-1)=15 \text { degrés de liberté. }
\end{aligned}
$$

TABLE V. - Distribution de larves de Sericostoma personatum : total des récoltes de chaque stade à chaque station (valeurs théoriques pour l'analyse de $\chi^{2}$ entre parenthèses $(7,68)$ etc...

\begin{tabular}{lccccccc}
\hline Stade & 1 & 2 & 3 & 4 & 5 & 6 & Total \\
\hline & 26 & 58 & 56 & 44 & 35 & 40 & 259 \\
Station 1 & $(7,68)$ & $(28,87)$ & $(45,02)$ & $(36,81)$ & $(49,26)$ & $(91,37)$ & \\
& 2 & 12 & 39 & 27 & 38 & 30 & 148 \\
Station 2 & $(4,39)$ & $(\mathbf{1 6 , 5 0 )}$ & $(25,73)$ & $(21,03)$ & $(28,15)$ & $(52,21)$ & \\
& 1 & 30 & 54 & 56 & 67 & 193 & 401 \\
Station 3 & $(11,89)$ & $(44,69)$ & $(69,70)$ & $(56,99)$ & $(76,26)$ & $(141,46)$ & \\
& 0 & 9 & 21 & 12 & 46 & 82 & 170 \\
Station 4 & $(5,04)$ & $(18,95)$ & $(29,55)$ & $(24,16)$ & $(32,33)$ & $(59,97)$ & \\
\hline Total & 29 & 109 & 170 & 139 & 186 & 345 & 978 \\
\hline
\end{tabular}

Ce $\chi^{2}$ étant bien au-dessus de la valeur $5 \%$ de la table II et de la valeur 37,70 à $0,1 \%$ de la table 8 de Pearson et Hartley (1966), l'association entre les deux classifications est hautement significative $(\mathrm{P}<0,001)$. Par suite, $\mathrm{H}_{0}$ est rejetée au seuil $0,1 \%$. La valeur $\mathrm{du} \chi^{2}$ pour chaque case indique que les stades 1 et 2 sont plus abondants que les valeurs théoriques à la station 1 et moins abondants aux stations 3 et 4 , alors que l'inverse prévaut pour les stades 5 et 6 .

\section{QUELQUES PROBLEMES POSES PAR L'ECHANTILLONNAGE SUR LE TERRAIN}

\section{ET LE SOUS-ECHANTILLONNAGE AU LABORATOIRE}

L'échantillonnage sur le terrain pose trois problèmes majeurs : (1) la dimension des relevés (ou unités d'échantillonnage) c'est-àdire la taille des quadrats, (2) le nombre de relevés dans l'aire étudiée, (3) la localisation des relevés dans l'aire étudiéc. 


\section{(1) Dimension des relevés.}

Des quadrats de petite taille conviennent mieux à l'étude de la dispersion d'une Population et, à ce sujet, les problèmes liés à la distinction des distributions non-au hasard ont été traités au paragraphe 3.1. Si une Population est vraiment distribuée au hasard, toute taille de quadrat est également efficace pour estimer les paramètres de population. Cette efficacilé est définie en comparant l'importance relative des échantillonnages pour des estimations de même précision. Plusieurs auteurs (par exemple Beall 1939, Finney 1946, Taylor 1953) ont étudié l'influence de la dimension des relevés sur l'efficacité de l'échantillonnage; leur conclusion est qu'un petit relevé est plus efficace qu'un grand lorsque la dispersion d'une Population est de type contagieux. En fait, les petits relevés présentent plusieurs avantages : (1) un plus grand nombre peut être étudié pour un même effort de traitement : tri, comptage, détermination; (2) avec plus de relevés, le nombre de degrés de liberté augmente, ce qui permet de réduire l'erreur statistique; (3) plusieurs petits relevés couvrent une plus grande partie de l'habitat que quelques grands, d'où un échantillonnage plus représentatif. En général, plus la dimension des relevés est petite, plus les résultats sont précis et représentatifs.

Bien que la solution idéale soit d'utiliser des relevés aussi petits que possible, des facteurs pratiques fixent une limite inférieure aux dimensions de ces relevés, par exemple la taille des pierres sur substrat pierreux. De plus, l'erreur d'échantillonnage faite sur les bords du relevé est proportionnellement plus grande pour un petit relevé. Par suite, le choix de la taille du quadrat est toujours un compromis entre des exigences statistiques et pratiques.

\section{(2) Nombre de relevés par échantillon.}

La dispersion de beaucoup d'espèces étant souvent de type contagieux, les Populations naturelles présentent de grandes variations et les petits échantillons (c'est-à-dire avec peu de relevés) sont statistiquement inexacts. Par suite, les nombres publiés sont souvent illusoires parce que les échantillons sont trop petits. La solution la plus simple serait de toujours prendre de grands échantillons, c'est-à-dire plus de 50 relevés par échantillon $(n>50)$. Malheureusement, il est habituellement impossible de trier et dénombrer toutes les espèces d'un très grand échantillon, notamment lorsque les échantillons sont fréquents. Un compromis doit donc être adopté entre la précision statistique et le travail d'examen des espèces. 
Une méthode simple peut être utilisée pour trouver le nombre convenable de relevés. Prendre 5 relevés au hasard et calculer la moyenne arithmétique. Prendre ensuite 5 relevés supplémentaires au hasard et calculer la moyenne pour 10 relevés. Continuer à augmenter la taille de l'échantillon par 5 relevés et placer sur un graphique les moyennes pour 5,1015 , etc., relevés par rapport à la taille de l'échantillon. Quand la moyenne devient stable, une dimension d'échantillon convenable est atteinte et peut être utilisée pour la station étudiée. Comme il est souvent impossible de calculer des moyennes au moment de l'échantillonnage, cette méthode simple n'a qu'une portée limitée.

Le nombre approché de relevés/échantillon peut aussi être calculé pour un degré de précision voulu (D) :

$$
\mathrm{n}=\frac{\mathrm{s}^{2}}{\mathrm{D}^{2} \overline{\mathrm{x}^{2}}}
$$

où $\mathrm{D}$ est l'erreur-standard demandée sous la forme d'une proportion de la moyenne (par exemple si l'on peut tolérer une erreurstandard égale aux $20 \%$ de la moyenne, on a $\mathrm{D}=0,2$ ce qui correspond à une erreur raisonnable dans la plupart des échantillons de fond). Les valeurs approchées de $\bar{x}$ et $s^{2}$ sont estimées à partir d'échantillons antérieurs ou préliminaires. Des formules spéciales existent pour les lois de Poisson et binomiale négative quand l'une ou l'autre de ces distributions est un modèle convenable des échantillons (voir Elliott 1971, chapitre 8).

\section{(3) Localisation des relevés dans l'aire étudiée.}

L'aire étudiée est l'ensemble de l'aire utilisable pour l'échantillonnage. Dans cette aire, le nombre de relevés possible est donné $\operatorname{par} \mathbf{N}=\mathbf{A}$ /a où $\mathbf{A}$ est l'aire étudiée et a l'aire d'un relevé (= taille du quadrat). $\mathrm{N}$ relevés forment done la population d'où un échantillon de $n$ relevés est extrait. $n$ étant généralement beaucoup plus petit que $\mathbf{N}$, il convient de choisir le mode de sélection du petit échantillon de $\mathrm{n}$ relevés à partir de la grande population de $\mathbf{N}$ relevés. Cet échantillon doit être représentatif de l'ensemble de la population, et les relevés doivent donc être choisis sans «biais». Ces conditions sont remplies quand les relevés sont extraits au hasard dans la population. Dans un échantillonnage au hasard simple, tous les relevés ont une chance égale d'être choisis. Une vraie sélection au hasard est souvent difficile à réaliser. La méthode la plus sûre est d'utiliser une table de nombres au hasard. Ces tables sont incluses dans beaucoup de manuels de statistiques, 
- une grande table est donnée par Fisher et Yates (1963, table 33). Pour l'utilisation de ces tables, sélectionner les n premiers nombres aux valeurs inférieures à $\mathbf{N}$. Si tous les relevés possibles de la population sont rangés de 1 à $\mathrm{N}$, les $\mathrm{n}$ relevés de chaque échantillon sont facilement localisés. Celte méthode est souvent pénible dans le cas d'une grande population. Il est plus facile d'utiliser une grande grille à deux dimensions dans laquelle chaque carré correspond à l'aire d'un relevé. Certains carrés de la grille sont en surnombre quand l'aire étudiée est irrégulière. Les carrés de deux côtés adjacents de la grille sont numérotés, et chaque relevé est ainsi localisé par ses coordonnées. Des nombres au hasard sont ensuite tirés par paires, et utilisés comme coordonnées pour localiser chaque relevé de l'échan!illon. Pour cela, on peut simplement placer deux lignes graduées à angle droit; on procède par arpentage quand l'aire étudiée est grande.

Une fois choisis au hasard, presque tous les relevés d'un échantillon peuvent tomber dans une même partie de l'aire étudiée. Un échantillonnage au hasard simple n'est donc pas très efficace, notamment quand $n$ est beaucoup plus petit que N. Par suite, la méthode plus efficace «d'échantillonnage au hasard stratifié » est toujours préférable à l'échantillonnage au hasard simple. L'échantillonnage stratifié augmente l'efficacité de l'échantillonnage en divisant la population en plusieurs sous-populations ou strates. Ces strates doivent être plus homogènes que l'ensemble de la population, et former des aires bien définies, de taille connue. La précision des estimations est ainsi augmentée, et les subdivisions de la population plus justement représentées au sein de l'échantillon. Les données des différentes strates peuvent être comparées dans une analyse de variance simple (sources de variation : entre les strates, à l'intérieur des strates).

Dans la forme la plus simple de stratification, l'aire étudiée est divisée en aires de même taille (= strates). Les relevés de l'échantillon sont également répartis par strates et localisés au hasard dans chaque strate. Si les strates ont des aires inégales, les relevés de l'échantillon sont inégalement répartis par strates, proportionnellement à l'aire de chaque strate, c'est-à-dire au nombre total de relevés possibles dans chaque strate. Par ce procédé, la fraction échantillonnée de chaque strate est la même, c'est-à-dire :

$$
\frac{\mathrm{n}_{1}}{\mathrm{~N}_{1}}=\frac{\mathrm{n}_{2}}{\mathrm{~N}_{2}}=\ldots \frac{\mathrm{n}_{\mathrm{k}}}{\mathrm{N}_{\mathrm{x}}}=\frac{\mathrm{n}}{\mathrm{N}}
$$

où l'on choisit des sous-échantillons pris au hasard de $n_{1}, n_{2} \ldots n_{k}$ relevés dans $k$ strates contenant $N_{1}, N_{2}, \ldots N_{k}$ relevés. L'échantillon est ainsi «pondéré » et sa moyenne arithmétique est la meil- 
leure estimation de la moyenne de la population. Le calcul est fait de la manière habituelle ou à partir des moyennes des $\mathrm{k}$ strates :

$$
x=\frac{\Sigma x}{n}=\frac{n_{1} \bar{x}_{1}+n_{2} \bar{x}_{2}+\ldots+n_{k} \bar{x}_{k}}{n}
$$

où $\overline{\mathbf{x}}_{1}, \overline{\mathrm{x}}_{2} \ldots \overline{\mathrm{x}}_{\mathrm{k}}$ sont les moyennes arithmétiques des différentes strates.

L'erreur-standard de la moyenne est :

$$
\frac{1}{\mathrm{n}} \sqrt{\mathrm{n}_{1} \mathrm{~s}^{2}{ }_{1}+\mathrm{n}_{2} \mathrm{~s}^{2}{ }_{2}+\ldots \mathrm{n}_{\mathrm{k}} \mathrm{s}^{2}{ }_{\mathrm{k}}}
$$

où $\mathrm{s}^{2}{ }_{1}, \mathrm{~s}_{2}{ }_{2}, \ldots \mathrm{s}^{2}{ }_{\mathrm{k}}$ sont les variances des différentes strates. Si la fraction échantillonnée excède $10 \%(\mathrm{n} / \mathrm{N}>0,1)$, l'erreur-s!andard est :

$$
\frac{1}{n} \sqrt{\left(n_{1} s_{11}^{2}+n_{2} s_{2}^{2}+\ldots+n_{k} s_{k}^{2}\right)(1-n / N)}
$$

où $\left(1-\frac{\mathrm{n}}{\mathrm{N}}\right)$ est la «correction de population finie».

Bien que la répartition proportionnelle soit la plus fréquente dans l'échantillonnage stratifié, la répartition optimale théorique des relevés dans l'échantillon est celle qui minimise l'erreurstandard de la moyenne estimée pour un effort d'échantillonnage donné. On y arrive quand la fraction échantillonnée pour chaque strate est proportionnelle à l'écart-lype pour cette strate. Les écarts-types des strates étant rarement connus avant l'échantillonnage, la méthode de répartition optimale est rarement utilisée. Avec une répartition optimale, l'échantillon n'est plus «pondéré 》 et par suite, une moyenne pondérée et une erreur-standard doivent être calculées (voir Snedecor et Cochran 1967).

L'échantillonnage stratifié est du plus grand intérêt quand l'aire étudiée comprend des biotopes variés. Par exemple, nous souhaitons échantillonner une section de rivière d'une aire égale à $200 \mathrm{~m}^{2}$, l'aire du relevé étant $0,05 \mathrm{~m}^{2}$. La population comprend donc 4000 relevés $(N=4000)$ d'où un échantillon au hasard de 40 relevés doit être extrait $(n=40)$. Les cinq strates suivantes $(k=5)$ ont été reconnues (entre parenthèses : nombre de relevés possibles dans chaque strate): grandes pierres sur cailloux $\left(N_{1}=1000\right)$, cailloux $\left(N_{2}=500\right)$, végétal A sur cailloux $\left(N_{3}=1500\right)$, végétal $B$ sur cailloux $\left(N_{4}=800\right)$, vase $\left(N_{i 5}=200\right)$. Les écarts- 
types des strates n'étant pas connus, la répartition optimale ne peut pas être faite. Les relevés de l'échantillon au hasard $(n=40)$ sont donc répartis proportionnellement aux aires des strates :

$$
\mathrm{n}_{1}=10, \mathrm{n}_{2}=5, \mathrm{n}_{3}=15, \mathrm{n}_{4}=8, \mathrm{n}_{5}=2 \text {. }
$$

Ces relevés sont choisis au hasard à partir des relevés possibles dans chaque strate. On notera que la fraction échantillonnée est la même pour toutes les strates $(\mathrm{n} / \mathrm{N}=\mathbf{0 , 0 1})$.

D'autres méthodes pour choisir un échantillon à partir d'une population sont brièvement revues par Elliott (1971, chapitre 8).

\section{(4) Sous-échantillonnage de récoltes abondantes au laboratoire.}

Le dénombrement des invertébrés présents dans un relevé devient un travail fastidieux quand les récoltes sont très abondantes. La réduction de la taille du relevé est souvent impossible. Il peut alors être procédé au "sous-échantillonnage» (ou "échantillonnage en deux stades $»)$. Dans un premier stade, un échantillon de $\mathbf{n}_{1}$ « relevés primaires» est pris au hasard dans la population sur le terrain. Dans un second stade, un sous-échantillon de $\mathrm{n}_{2}$ 《sous-échantillons 》 (ou « relevés de second stade ») est pris à partir de chaque relevé primaire. Pour cela, le contenu de chaque relevé primaire est concentré dans un volume d'eau (ou de mélange conservateur) connu, et bien agité dans un récipient avant le sous-échantillonnage. Si les invertébrés sont distribués au hasard dans le récipient avant le sous-échantillonnage, et si une petite proportion seulement de la prise totale est enlevée dans chaque sous-relevé, les nombres de chaque sous-échantillon sont distribués selon une loi de Poisson. Cette hypothèse doit être vérifiée par un test $\chi^{2}$ (rapport variance à la moyenne, $\S$ 3.1.1.) sur un sous-échantillon d'au moins cinq relevés. Si l'accord avec une loi de Poisson est accepté, on peut supposer que les invertébrés sont distribués au hasard avant le sous-échantillonnage; en conséquence il suffit d'extraire un sous-échantillon d'un sous-relevé seulement. Ainsi, un simple dénombrement peut être utilisé pour estimer les nombres totaux de chaque relevé primaire; et la précision de cette estimation dépend du nombre d'invertébrés dénombrés (§ 4.2.).

Si les nombres d'un sous-échantillon ne suivent pas une Ioi de Poisson, et si la variance $d u$ sous-échantillon est significativement plus grande que la moyenne, les invertébrés étaient distribués selon le type contagieux avant le sous-échantillonnage. Il est alors plus difficile d'estimer les nombres totaux et les limites de confiance pour chaque relevé primaire. La méthode la plus simple est de transformer les nombres d'un relevé en logarithmes, puis 
de calculer la moyenne géométrique avec ses limites de confiance à $95 \%$ (voir $\S$ 4.3.).

Le sous-échantillonnage est largement utilisé dans l'étude quantitative des Populations de plancton, et plusieurs articles exposent en détail les méthodes statistiques s'y rapportant (par exemple Ricker 1937, Holmes et Widrig 1956, Kutkuhn 1958, Lund, Kipling et Le Cren 1958).

\section{TRAVAOX CITES}

Albrecht (M. L.). 1959. - Die quantitative Untersuchung der Bodenfauna fliessender Gewässer. Z. Fishch., 8 : 481-550.

BAILey (N. T. J.). 1959. - Statistical Methods in Biology. London, 200 p.

Beall (G.). 1939. - Methods of estimating the population of insects in a field. Biometrika, $30: 422-439$.

Bertrand (H.). 1954. - Les insectes aquatiques d'Europe, t. I et II. Paris, 556 p. +547 p.

Bonnet (L.). 1964. - Le peuplement thécamoebien des sols. Rev. Ecol. Biol. Sol, 1 : 123-408.

Campbell (R. C.). 1967. - Statistics for Biologists. Cambridge, 242 p.

Cochran (W. G.) 1954. - Some methods for strengthening the common $\chi^{2}$ tests. Biometrics, $10: 417-451$.

Crow (E. L.) et Gardner (R. S.). 1959. - Table of confidence limits for the expectation of a Poisson variable. Biometrika, 46:441-453 (New Statistical Tables Series, $N^{\circ} 28$ ).

Cummins (K.W.). 1962. - An evaluation of some techniques for the collection and analysis of benthic samples with special emphasis on lotic waters. Am. Midl. Nat., 67 : 477-504.

Edgar (W. D.) et Meadows (P. S.). 1969. - Case construction, movement, spatial distribution and substrata selection in the larva of Chironomus riparius Meigen. J. exp. Biol., 50 : 247-253.

Edmondson (W.T.) et Winberg (G. G.). 1971. - A manual on methods for the assessment of secondary productivity in fresh waters. I.B.P. Handbook $n^{\circ}$ 17. Oxford, Blackwell, $358 \mathrm{p}$.

Elliotr (J. M.). 1971. - Some methods for the statistical analysis of samples of benthic invertebrates. Scient. Publs Freshwat. biol. Ass., 25, 144 p.

Finney (D. J.). 1946. - Field sampling for the estimation of wireworn populations. Biometrics, 2 (1) : 1-7.

Fisher (R. A.) et Yates (F.). 1963. - Statistical Tables for Biological, Agricultural and Medical Research (6th ed.). Edinburgh, $146 \mathrm{p}$.

Gaufin (A. R.), Harris (E. K.) et Walker (H. J.). 1956. - A statistical evaluation of bottom sampling data obtained from three standard samplers. Ecology, 37 : 643-648.

Greig-Smith (P.). 1964. - Quantitative Plant Ecology (2nd ed.). London, 256 p.

Harris (E. K.). 1957. - Further results in the statistical analysis of stream sampling. Ecology, $38: 463-468$.

HeYwood (J.) et Edwards (R. W.). 1961. - Some aspects of the ecology of Potamopyrgus jenkinsi Smith. J. Anim. Ecol., 31 : 239-250. 
Holmes (R. W.) et Widrig (T. M.). 1956. - The enumeration and collection of marine phytoplankton. J. Cons. perm. int. Explor. Mer., $22: 21-32$.

KutkuhN (J.H.). 1958. - Notes on the precision of numerical and volumetric plankton estimates from small-sample concentrates. Limnol. Oceanogr., $3: 69-83$.

LAMotTE (M.). 1971. - Initiation aux méthodes statistiques en biolngie (2 éd.). Paris, 144 p.

LAMotTE (M.) et Bourlí̀re (F.) (éd.). 1971. - L'échantillonnage des peuplements animaux des milieux aquatiques. Paris, $294 \mathrm{p}$.

Lison (L.). 1958. - Statistique appliquée à la biologie expérimentale. La planification de l'expérience et l'analyse des résultats. Paris, $346 \mathrm{p}$.

Lund (J. W. G.), Kipling (C.) et Le Cren (E. D.). 1958. - The inverted microscope method of estimating algal numbers and the statistical basis of estimations by counting. Hydrobiologia, $11: 143-170$.

Macan (T. T.). 1958. - Methods of sampling the bottom fauna in stony streams. Mitt. int. Verein. theor, angew. Limnol., $8: 1-21$.

Macan (T. T.). 1959. - A guide to Freshwater Invertebrate Animals. London, $118 \mathrm{p}$.

Pearson (E. S.) et Hartiey (H. O.). 1966. - Biometrika Tables for Statisticians (3rd ed). Cambridge, 264 p.

Ricker (W. E.). 1937. - Statistical treatment of sampling processes useful in the enumeration of plankton organisms. Arch. Hydrobiol., $31: 68-84$.

Schwoerbel (J.). 1970. - Methods of hydrobiology (freshwater biology). Oxford, Pergamon, Translated from « Methoden der Hydrobiologie $\gg, 200 \mathrm{p}$.

SiEger. (S.). 1956. - Non-parametric Statistics for the Behavioral Sciences. New York, 312 p.

Snedecor (G. W.) et Cochrin (W. G.). 1967. - Statistical Methods. Ames, Iowa, 593 p.

Southwood (T. R. E.). 1966. - Ecological Methods. London, 391 p.

TAYloR (C. C.). 1953. - Nature of variability in trawl catches. Fishery Bull. Fish Wildl. Serv. U.S., 54 : 145-166.

Vesserkau (A.). 1960. - Methodes statistiques en biologie et en agronomie. Paris, 539 p.

Welch (P. S.). 1948. - Limnological Methods. Philadelphia, 381 p.

Wilcoxon (F.) et Wilcox (R. A.). 1964. - Some Rapid Approximate Statistical Procedures. New York, $60 \mathrm{p}$. 